\title{
A POLYHEDRAL CHARACTERIZATION OF BORDER BASES
}

\author{
GÁBOR BRAUN AND SEBASTIAN POKUTTA
}

\begin{abstract}
Aвstract. Border bases arise as a canonical generalization of Gröbner bases, using order ideals instead of term orderings. We provide a polyhedral characterization of all order ideals (and hence all border bases) that are supported by a zero-dimensional ideal: order ideals that support a border basis correspond one-to-one to integral points of the order ideal polytope. In particular, we establish a crucial connection between the ideal and its combinatorial structure. Based on this characterization we also provide an adaptation of the border basis algorithm of Kehrein and Kreuzer [35] to allow for computing border bases for arbitrary order ideals, given implicitly via maximizing a preference on monomials (variable selection problem), independent of term orderings. The algorithm requires the same size of resources as the border basis algorithm except for some minor overhead. We also show that the underlying variable selection problem of finding an order ideal that supports a border basis is NP-hard and that any linear description of the associated convex hull of all order ideals requires a superpolynomial number of inequalities.
\end{abstract}

\section{INTRODUCTION}

In many different disciplines and real-world applications one is faced with solving systems of polynomial equations. Often this is simply due to a physical or dynamical system having a natural representation as a system of polynomial equations, but equally often it is due to the sheer expressive power of polynomial systems of equations that allow for easy reformulation. To give an example of the latter, an inequality $a x \leq b$ with $a \in \mathbb{R}^{n}$ and $b \in \mathbb{R}$ can be expressed via a single polynomial equation: $a x+u^{2}=b$. A slightly more involved example is that of the feasible region of a binary program $\left\{x \mid A x \leq b, x \in\{0,1\}^{n}\right\}$, which can be captured via rewriting each individual inequality as before, and adding quadratic polynomials $x_{i}^{2}-x_{i}=0$ for each coordinate $i=1, \ldots, n$ of $x$. As a consequence, there is a huge need to computationally model, understand, manipulate, and extract the solution set of systems of polynomial equations.

A key insight in (computational) commutative algebra is that one can choose a smart ordering on the monomials and compute a special set of generators of the ideal generated by the system of equations that makes many operations easy, and provides a structural insight into the system. One such special set of generators is a Gröbner basis. By now, Gröbner bases are fundamental and standard tools in commutative algebra to actually perform important operations on ideals such as intersection, membership test, elimination, projection, and many more. Border bases arise as a natural generalization of Gröbner bases that can be computed for zero-dimensional ideals, i.e., the associated factor space is a finite-dimensional vector space (see Section 2 for details). While this might seem to be a severe restriction, for many applications it is sufficient. Roughly speaking, whenever the solution set is finite, we are dealing with a zero-dimensional ideal. For example, systems of polynomial equations with solutions restricted to a finite set of points are captured by zero-dimesional ideals.

Date: September 13, 2021.

2000 Mathematics Subject Classification. Primary: 13P10; 90C57; secondary: 65H10;12Y05; 90C27; $68 \mathrm{R} 05$.

Key words and phrases. order ideal polytope, border bases, Gröbner bases, combinatorial optimization.

Research partially supported by German Research Foundation (DFG) funded SFB 805, Hungarian Scientific Research Fund, grant No. K 67928, NSF grants CMMI-1333789 and CCF-1415496. 
The advantage of border bases over Gröbner bases partly arises from the iterative generation of linear syzygies, inherent in the border basis algorithm, which allows for successively approximating the basis of interest degree-by-degree, which leads to an implied notion of approximability. Moreover, many border basis algorithms (and also Gröbner basis algorithms) are essentially linear algebra algorithms, allowing for fast computation. However arguably the most important difference between a Gröbner basis and a border basis is that the former is computed with respect to a term ordering (the aforementioned ordering on the monomials) and the latter is computed with respect to a so-called order ideal, which for now can be thought of as a generalization of a term ordering (and not an ideal in the usual sense). An order ideal that supports a border basis, i.e., for which one can compute a border basis is called admissible. It is well known that every reduced Gröbner basis can be extended to a border basis (see [35, p. 281ff]), i.e., every term ordering gives rise to an admissible order ideal. At the same time, not every border basis is an extension of a Gröbner basis, since the former form a strictly larger set giving potential extra freedom in modeling solution sets to polynomial systems. For a given zero-dimensional ideal $I$ of a polynomial ring $R$, the size of an order ideal that supports a border basis is predetermined as the dimension of the vector space $R / I$. This is only a necessary condition though and not every order ideal $\mathcal{O}$ of size equal to the dimension of $R / I$ supports a border basis. An example illustrating these two cases is presented in [35, Example 6]. Finally, border bases deform more smoothly in the input [40] (see also [49]), which is particularly helpful when the coefficients arise from measurement data [1,29], e.g., that is why border bases are used in the context of total-least-squares polynomial regression (see [29] for details).

1.1. Arbitrary order ideals. While the above examples highlight the advantages of border bases for certain types of computations, so far we have not answered a key question: why it is desirable and important to be able to compute border bases with respect to general order ideals (i.e., those that do not necessarily stem from a term ordering). We offer three different perspectives.

First, it is desirable to obtain a complete characterization of all border bases supporting order ideals. In particular, our characterization can be used to rule out certain types of order ideals and provides a proof (a dual certificate) for their non-existence via the associated violated inequality: in complexity-theoretic terms, we provide certificates for the non-membership problem. Second, choosing a different order ideal might significantly reduce computational time to obtain a border basis. This aspect is well known and often exploited in the context of Gröbner bases.

However, arguably the most important aspect from the perspective of actually solving polynomial system is variable selection. Often the polynomial system of interest stems from e.g., a physical system and the variables correspond to actual physical quantities and hence have explanatory power. Now, it can be very desirable to obtain a polynomial description of the solution set of the system using specific monomials or variables to allow for actual real-world interpretation of the solutions. At the same time the ideal structure might preclude a description with all desired variables or monomials contained in the order ideal. We end up with an optimization problem of finding an order ideal, which matches our preferences as well as possible. Optimization problems of this type are referred to as variable selection or feature selection (see e.g, [31]) and are ubiquitous in many data related disciplines, such as e.g., statistics, machine learning, and more broadly data analytics, where we effectively seek an explanation of a phenomenon in specified explanatory variables. In our context this naturally leads to the MAXIMUM WEIGHT ADMISSIBLE ORDER IDEAL problem, where we specify weights for each monomial and we search a maximal weight order ideal supporting a border basis of a given zero-dimensional ideal $I$.

Order ideals and determining those with maximum weight do appear in a very natural way in combinatorial optimization as the so called maximum weight closure problem (a simplified version of our MAXIMUM WEIGHT ADMISSIBLE ORDER IDEAL problem, cf. e.g., [47]) and they have a variety of applications, e.g., in open-pit mining where any feasible production plan is indeed an order ideal; 
we refer the interested reader to [30] for an overview. Another example is the approximate vanishing ideal algorithm in [29], which computes a polynomial description of an approximate vanishing ideal of a given set of (noisy) points. Effectively, a total-least-squares optimization problem is solved here and explanatory variables can come from an order ideal. If the points stem from actual (physical) measurements, choosing the variables in the order ideal can help recover important physical relations. Other, more involved applications might arise, e.g., in computational biology where the structure of a boolean network is inferred from the Gröbner fan.

It has been an open question to characterize the admissible order ideals of a zero-dimensional ideal. We provide a polyhedral relaxation of all admissible order ideals of a given zero-dimensional ideal that support a border basis. This is the best we can hope for given that the separation problem for the polytope is NP-hard as we will see in Section 5. Moreover, we will also establish that in general the convex hull of all order ideals of a given ideal can require a superpolynomial number of inequalities in any linear programming formulation, i.e., that the polytope arising from the convex hull has superpolynomial extension complexity. Many of the results that we present later are the border bases analogs of their counterpart for Gröbner bases in [46].

1.2. Computing border bases. The border basis algorithm in [35], which is a specification of Mourrain's generic algorithm [43], allows for computing border bases of zero-dimensional ideals for order ideals supported by a degree-compatible term ordering. However, this border basis algorithm does not allow for the computation of a border basis for more general order ideals (in fact it requires a degree-compatible term ordering). The alternative algorithm presented in [35, Proposition 5] which can potentially compute arbitrary border bases requires the a priori knowledge of the order ideal that might support a border basis. So while in principle the algorithm can compute arbitrary border bases, the supporting order ideal has to be part of the input. Thus it does not characterize order ideals for which a border basis does exist. Further, as pointed out in [35, p. 284], the basis transformation approach of this algorithm is unsatisfactory as it significantly relies on Gröbner basis computations. Another interesting approach for the computation of normal forms that do not require degree-compatible term orderings is [44, 45], however here a fixpoint scheme is required.

1.3. Applications of border bases. Surprisingly, it turns out that there are deep connections to other mathematical disciplines and border bases represent the combinatorial structure of the ideal under consideration in a canonical way. Although the use of border basis as a concise framework is quite recent (see e.g., [34-36]), the concept of border bases and in particular the border basis algorithm is rather old and has been reinvented in different fields of mathematics including computer algebra, discrete optimization, logic, and cryptography under different names. In summary, border bases have been successfully used for solving zero-dimensional systems of polynomial equations (see, e.g., [8,42, 43]), which in particular include those with $0 / 1$ solutions and thus a large variety of combinatorial problems.

Polynomial method. Polynomial systems have been used in discrete mathematics and combinatorial optimization to formulate combinatorial problems such as the graph coloring problem, the stable set problem, and the matching problem (we refer the interested reader to [22]) as well as to recognize graph properties [24]. This well-known method, which Alon referred to as the polynomial method [3, 4] recently regained strong interest and emphasizes the alternative view of border bases algorithms in their various incarnations as proof systems which successively uncovers hidden information by making it explicit. In [23, Section 2.3] and [21, 22, 25] infeasibility of certain combinatorial problems, e.g., 3-colorability of graphs is established using Hilbert's Nullstellensatz and the algorithm NulLA is provided to establish infeasibility by using a linear relaxation. The core of the algorithm is identical to the $L$-stable span procedure used in the border basis algorithm in [35], which intimately links both procedures. The difference is of a technical but important 
nature: whereas NuILA establishes infeasibility, the border basis algorithm as presented in [35] computes the actual border bases of the ideal. Another recent link between border bases and the Sherali-Adams closure [51] is that the Sherali-Adams procedure can be understood as a weaker version of the $L$-stable span procedure, see [48]. Recently, border bases have also been used to obtain a hierarchy of relaxations for polynomial optimization problems [14].

Border bases and cryptography. Border bases have also been used to solve sparse quadratic systems of equations thus giving rise to applications in cryptography in a natural way. Such systems arise from crypto systems (such as e.g., AES, BES, HFE, DES, CTC variants) when rewriting the S-boxes as polynomial equations. The celebrated XL, XSL, MutantXL attacks (see e.g., [17,41]) are equivalent to the reformulation-linearization-technique (RLT) of Sherali and Adams [51] and use a version of the Nullstellensatz to break ciphers. Motivated by the success of the aforementioned methods, border bases have also been used in cryptanalysis and coding theory, see [9].

Border bases and numerical computations. Another core application of border bases is the modeling of dynamic systems from measured data (see e.g., [1, 29, 37]) where better numerical stability is advantageous. The obtained solutions via border bases often provide a better generalization, i.e., explain new phenomena better, than the respective Gröbner basis analog.

Our contribution. Our contribution is the following:

Polyhedral characterization of all border bases. We provide a complete, polyhedral characterization of all border bases of any zero-dimensional ideal $I$. We associate an order ideal polytope $P$ to $I$ whose integral points are in one-to-one correspondence with order ideals supporting a border basis of $I$ (Theorem 3.2). This explicitly establishes the link between the combinatorial structure of the basis of the factor space and the structure of the ideal: whether an order ideal supports a border basis is solely determined by the combinatorial structure of the order ideal polytope. A related result for Gröbner bases of the vanishing ideal of generic points was established in [46], where it was shown that distinct reduced Gröbner bases of the vanishing ideal are in bijection with the vertices of the corner cut polyhedron.

Computing maximum weight order ideals. We will show that computing a maximum weight order ideal supporting a border basis, i.e., solving the variable selection problem, is NP-hard in general (Theorem [5.1). This is surprising as we merely ask for a basis transformation. In particular, the NP-hardness does not stem from the hardness of computing the L-stabilized span, as the problem remains NP-hard, even in cases where the $L$-stabilized span is small enough to be determined efficiently as shown in our reduction in Section 5.2. In particular, unless NP $=$ coNP, the convex hull of characteristic vectors of order ideals cannot have an efficient linear programming formulation. In Section 5.3, we complement this result and show that there exists zero-dimensional ideals so that any linear programming formulation capturing their admissible order ideals requires a subexponential number of inequalities, irrespective of NP vs. coNP; in the language of extended formulations, we show that the convex hull of admissible order ideals has subexponential extension complexity. We discuss implications of this in Section 5.4.

Computing arbitrary border bases. We extend the border basis algorithm in [35] to compute border bases for arbitrary order ideals using the order ideal polytope (Algorithm 4.1), where the order ideals are given implicitly by a preference vector. (Note that every admissible order ideal can be obtained by choosing a suitable preference vector.) We would like to point out that algorithms for general bases of quotient spaces have been proposed in [44, 45]). However these algorithms are markedly different in relying on some fix point scheme, so that the advantage of the degree-bydegree iterations are lost. We refer the reader to the discussion in [33]. 
Computational feasibility. We provide computational tests that demonstrate the feasibility of our method. Having the order ideal polytope available for a zero-dimensional ideal $I$, it is possible to examine the structure of the ideal based on its border bases. To demonstrate feasibility, we consider the straightforward application of counting the number of border bases for a zero-dimensional ideal I, which we present as an example in Section 6 for counting degree-compatible order ideals.

Subsequent work. Following our work [11,12] several related results have been obtained. In [6] it was shown that the border basis detection problem is NP-hard (see also [5]). Moreover, an algorithm for computing border bases without term orderings has been given in [33].

Outline. We start with the necessary preliminaries in Section 2 and recall the border basis algorithm from [35] in Section 2.2. In[Section 3] we introduce the order ideal polytope and establish the one-to-one correspondence between the integral points of this polytope and border bases. We also derive an equivalent characterization that is better suited for actual computations. In Section 4 we then use the results from Section 3 to obtain the generalized border basis algorithm for arbitrary order ideals. We then study the complexity of the MAXIMUM WEIGHT ADMISSIBLE ORDER IDEAL problem establishing NP-hardness and a subexponential lower bound on its polyhedral complexity in Section 5. We conclude with computational results in Section 6 and with a summary in Section 7.

\section{Preliminaries}

We consider a polynomial ring $K[\mathbb{X}]$ over the field $K$ with variables $\mathbb{X}=\left\{x_{1}, \ldots, x_{n}\right\}$. Let $\mathbb{T}^{n}:=$ $\left\{\prod_{i} x_{i}^{k_{i}} \mid k_{i} \in N\right\}$ be the set of terms, i.e., the set of all monomials. Recall that the (total) degree of a monomial $m=\prod_{i} x_{i}^{k_{i}}$ is $\operatorname{deg} m=\sum_{i} k_{i}$. For any $d \in \mathbb{N}$ we let $\mathbb{T}_{\leq d}^{n}:=\left\{m \in \mathbb{T}^{n} \mid \operatorname{deg} m \leq d\right\}$ be the set of monomials of total degree at most $d$. Sometimes we will refer to a subset of monomials $L$ as the computational universe, to which the actual computation is confined. For a polynomial $p=$ $\sum_{m \in \mathbb{T}^{n}} a_{m} m \in K[\mathbb{X}]$ we define the support of $p$ to be $\operatorname{supp}(p):=\left\{m \in \mathbb{T}^{n} \mid a_{m} \neq 0\right\}$ and similarly, for a set of polynomials $P \subseteq K[\mathbb{X}]$ we define the support of $P$ to be $\operatorname{supp}(P):=\bigcup_{p \in P} \operatorname{supp}(p)$. Given a (total) ordering $\sigma$ on $\mathbb{T}^{n}$, the leading term $\operatorname{LT}_{\sigma}(p):=m$ of the polynomial $p$ is the largest element $m$ of $\operatorname{supp}(p)$ in the ordering $\sigma$, and the leading coefficient $\operatorname{LC}_{\sigma}(p)=a_{m}$ of $p$ is the coefficient of $\operatorname{LT}_{\sigma}(p)$. We drop the index $\sigma$ if the ordering is clear from the context. Recall that a term ordering is a total ordering $\sigma$ on $\mathbb{T}^{n}$ with $m_{1} \leq m_{2}$ for all pair of monomials $m_{1}, m_{2}$ with $m_{1} \mid m_{2}$. Monomial orderings are used for Gröbner basis computations, but here we allow more general orderings.

The leading form $\operatorname{LF}(p)$ of a polynomial $p=\sum_{m \in \mathbb{T}^{n}} a_{m} m \in K[\mathbb{X}]$ is defined to be $\operatorname{LF}(p)=$ $\sum_{m: \operatorname{deg} m=\operatorname{deg} p} a_{m} m$, i.e., we single out the part with maximum degree. (The leading form does not depend on an ordering.) Both LF and LT generalize to sets in the obvious way, i.e., for a set of polynomials $P$ we define $\operatorname{LF}(P):=\{\operatorname{LF}(p) \mid p \in P\}$ and $\operatorname{LT}(P):=\{\operatorname{LT}(p) \mid p \in P\}$.

In the following we will frequently switch between considering polynomials $M$, the generated ideal, and the generated vector space whose coordinates are indexed by the monomials in the support of $M$. We denote the ideal generated by $M$ as $\langle M\rangle_{K[\mathbb{X}]}$ and the vector space generated by $M$ as $\langle M\rangle_{K}$. For $n \in \mathbb{N}$ we define $[n]:=\{1, \ldots, n\}$. All other notation is standard as to be found in [18, 38]; we have chosen the border basis specific notation to be similar to the one in [35]; see also [39] for a broader exposition.

2.1. Order ideals. Central to our discussion will be the notion of an order ideal, which is not an ideal, but a set of monomials closed under taking (monomial) factors:

Definition 2.1. Let $\mathcal{O}$ be a finite subset of $\mathbb{T}^{n}$. If for all $m \in \mathcal{O}$ and $m^{\prime} \in \mathbb{T}^{n}$ such that $m^{\prime} \mid m$ we have $m^{\prime} \in \mathcal{O}$, i.e., $\mathcal{O}$ is closed under factors, then we call $\mathcal{O}$ an order ideal. Furthermore, the border $\partial \mathcal{O}$ of a non-empty order ideal $\mathcal{O}$ is the set of monomials $\partial \mathcal{O}:=\left\{x_{j} m \mid j \in[n], m \in \mathcal{O}\right\} \backslash \mathcal{O}$. As an exception, we set $\partial \varnothing:=\{1\}$ for the empty order ideal. 
Examples of order ideals are $\{1\},\left\{1, x_{1}, \ldots, x_{1}^{k}\right\}$, and $\left\{1, x_{1}, x_{1}^{2}, x_{1} x_{2}, x_{2}\right\}$. Recall that an ideal $I \subseteq K[\mathbb{X}]$ is zero-dimensional, if and only if $K[\mathbb{X}] / I$ is finite dimensional. The $\mathcal{O}$-border basis of a zero-dimensional ideal $I$ is a special set of polynomials:

Definition 2.2. Let $\mathcal{O}=\left\{m_{1}, \ldots, m_{\mu}\right\}$ be an order ideal with border $\partial \mathcal{O}=\left\{b_{1}, \ldots, b_{v}\right\}$. Further let $I \subseteq K[\mathbb{X}]$ be a zero-dimensional ideal, and $\mathcal{G}=\left\{g_{1}, \ldots, g_{v}\right\} \subseteq I$ be a (finite) set of polynomials. Then the set $\mathcal{G}$ is an $\mathcal{O}$-border basis of $I$ if:

(1) the polynomials in $\mathcal{G}$ have the form $g_{j}=b_{j}-\sum_{i=1}^{\mu} \alpha_{i j} m_{i}$ for $j \in[v]$ and $\alpha_{i j} \in K$;

(2) $K[\mathbb{X}]=I \oplus\langle\mathcal{O}\rangle_{K}$ as vector spaces.

If there exists an $\mathcal{O}$-border basis of $I$ then the order ideal $\mathcal{O}$ supports a border basis of $I$, equivalently, $\mathcal{O}$ is admissible for $I$. Let $\Lambda(I)$ denote the set of admissible order ideals of $I$.

Note that any border basis $\mathcal{G}$ of an ideal $I$ is actually generating $I$ as an ideal, i.e. $\langle\mathcal{G}\rangle_{K[\mathbb{X}]}=I$. A proof of this fact can be found in [36. Proposition 4.3.2]; for the sake of completeness we provide an alternative proof here. Let us consider the subspace $\langle\mathcal{G}\rangle_{K[\mathbb{X}]}+\langle\mathcal{O}\rangle_{K}$ spanned by the ideal $\langle\mathcal{G}\rangle_{K[\mathbb{X}]}$ generated by $\mathcal{G}$ and the order ideal $\mathcal{O}$. This subspace is closed under multiplication by the $x_{i}$, and hence it is an ideal. As it contains 1 (being contained in either $\mathcal{G}$ or $\mathcal{O}$ ), it must be the whole ring, and hence $I=\left(\langle\mathcal{G}\rangle_{K[\mathbb{X}]}+\langle\mathcal{O}\rangle_{K}\right) \cap I=\langle\mathcal{G}\rangle_{K[\mathbb{X}]}+\left(\langle\mathcal{O}\rangle_{K} \cap I\right)=\langle\mathcal{G}\rangle_{K[\mathbb{X}]}$ by the modular law. Recall that the modular law states $(A+B) \cap C=A+(B \cap C)$ for all subspaces $A, B, C$ of a vector space with $A \subseteq C$.

In particular, an order ideal $\mathcal{O}$ supports an $\mathcal{O}$-border basis of $I$ if and only if $K[\mathbb{X}]=I \oplus\langle\mathcal{O}\rangle_{K}$. Moreover, for any given order ideal $\mathcal{O}$ and ideal $I$ the $\mathcal{O}$-border basis of $I$ is unique as $b_{j}$ has a unique representation in $K[\mathbb{X}]=I \oplus\langle\mathcal{O}\rangle_{K}$ for all $j \in[v]$. Furthermore, as $K[\mathbb{X}]=I \oplus\langle\mathcal{O}\rangle_{K}$ it follows that $|\mathcal{O}|=\operatorname{dim}\langle\mathcal{O}\rangle_{K}$ is invariant for all choices of $\mathcal{O}$. The requirement for $I$ being zero-dimensional is necessary to ensure finiteness of the order ideal $\mathcal{O}$ and its border $\partial \mathcal{O}$.

Example 2.3 (Order ideals from Gröbner bases). A common way to obtain an admissible order ideal $\mathcal{O}$ for a zero-dimensional ideal $I$ is to compute the Gröbner basis $\mathcal{G}=\left\{g_{1}, \ldots, g_{v}\right\} \subseteq K[\mathbb{X}]$ of $I$ with an arbitrary term ordering $\sigma$, and let

$$
\mathcal{O}:=\left\{m \in \mathbb{T} \mid \forall j: \operatorname{LT}_{\sigma}\left(g_{j}\right) \nmid m\right\}
$$

consists of all monomials $m$ not divisible by any leading term in the Gröbner basis.

As a concrete example, Tables 1 and 2 list every admissible order ideal of the following ideals over $K\left[x_{1}, x_{2}\right]$, indicating a term ordering providing them if any (the ground field $K$ can be any field):

$$
\begin{aligned}
& I_{1}:=\left\langle x_{1} x_{2}-x_{1}-x_{2}+1, x_{2}^{2}+x_{1}-1\right\rangle_{K[\mathbb{X}]} \\
& I_{2}:=\left\langle x_{1}^{2}-x_{1} x_{2}, x_{2}^{2}-x_{1} x_{2}, \mathbb{T}_{=3}^{2}\right\rangle_{K[\mathbb{X}]}
\end{aligned}
$$

We enumerate the order ideals of $I_{i}$ as $\mathcal{O}_{i, 1}, \mathcal{O}_{i, 2}, \ldots$ with the first index identifying the ideal $I_{i}$.

We leave it to the reader to verify that there is no further admissible order ideal for $I_{1}$ and $I_{2}$. (For $I_{1}$, Figure 3.1 showing linear dependence relations between monomials in $K\left[x_{1}, x_{2}\right] / I_{1}$ should be helpful.) In the case of $I_{2}$ the last admissible order ideal $\left\{1, x_{1}, x_{2}, x_{1} x_{2}\right\}$ does not come from any term ordering, as we will show now. First note that this order ideal is indeed admissible: a basis of $K\left[x_{1}, x_{2}\right] / I_{2}$ is given by the image of the admissible order ideal $\mathcal{O}_{2,2}=\left\{1, x_{1}, x_{2}, x_{1}^{2}\right\}$. As $x_{1}^{2}-x_{1} x_{2} \in I_{2}$, the image of $x_{1}^{2}$ is the same as the image of $x_{1} x_{2}$, thus the image of $\mathcal{O}_{2.3}=$ $\left\{1, x_{1}, x_{2}, x_{1} x_{2}\right\}$ coincides with that of $\mathcal{O}_{2,2}$, and hence it is a basis of $K\left[x_{1}, x_{2}\right] / I_{2}$. In particular, $K\left[x_{1}, x_{2}\right]=I_{2} \oplus\left\langle\mathcal{O}_{2,3}\right\rangle_{K}$ holds, showing the admissibility of $\mathcal{O}_{2,3}$.

If $\mathcal{O}_{2,3}$ came from a Gröbner basis $\mathcal{G}$ for a term ordering with $x_{1}>x_{2}$, then $\mathcal{G}$ would contain a $g \in \mathcal{G}$ whose leading term divides the leading term $x_{1} x_{2}$ of $x_{1} x_{2}-x_{2}^{2} \in I$, and therefore $\operatorname{LT}_{\sigma}(g) \in$ $\mathcal{O}_{2,3}$, a contradiction. The argument is similar for term orderings with $x_{1}<x_{2}$. 


\begin{tabular}{|c|c|c|c|}
\hline Term ordering & Gröbner basis & Admissible order ideal & Border basis \\
\hline lex & $\begin{array}{c}x_{1}+x_{2}^{2}-1 \\
x_{2}^{3}-x_{2}^{2}\end{array}$ & $\mathcal{O}_{1,1}=\left\{1, x_{2}, x_{2}^{2}\right\}$ & $\begin{array}{c}x_{1}+x_{2}^{2}-1, \\
x_{1} x_{2}+x_{2}^{2}-x_{2} \\
x_{1} x_{2}^{2}, x_{2}^{3}-x_{2}^{2}\end{array}$ \\
\hline degrevlex & $\begin{array}{c}x_{1}^{2}-x_{1} \\
x_{1} x_{2}-x_{1}-x_{2}+1 \\
x_{2}^{2}+x_{1}-1\end{array}$ & $\mathcal{O}_{1,2}=\left\{1, x_{1}, x_{2}\right\}$ & $\begin{array}{c}x_{1} x_{2}-x_{1}-x_{2}+1 \\
x_{1}^{2}-x_{1} \\
x_{2}^{2}+x_{1}-1\end{array}$ \\
\hline
\end{tabular}

TABLE 1. All admissible order ideals of $I_{1}=\left\langle x_{1} x_{2}-x_{1}-x_{2}+1, x_{2}^{2}+x_{1}-1\right\rangle_{K[\mathbb{X}]}$ over $K\left[x_{1}, x_{2}\right]$. Here $x_{1}>x_{2}$ in both term orderings.

\begin{tabular}{|c|c|l|c|}
\hline Term ordering & Gröbner basis & Admissible order ideal & Border basis \\
\hline \hline lex or degrevlex $\left(x_{1}>x_{2}\right)$ & $\begin{array}{c}x_{1}^{2}-x_{1} x_{2} \\
x_{1} x_{2}-x_{2}^{2}, x_{2}^{3}\end{array}$ & $\mathcal{O}_{2,1}=\left\{1, x_{1}, x_{2}, x_{2}^{2}\right\}$ & $\begin{array}{c}x_{1}^{2}-x_{2}^{2} \\
x_{1} x_{2}-x_{2}^{2} \\
x_{1} x_{2}^{2}, x_{2}^{3}\end{array}$ \\
\hline lex or degrevlex $\left(x_{2}>x_{1}\right)$ & $\begin{array}{c}x_{2}^{2}-x_{1} x_{2}, \\
x_{1} x_{2}-x_{1}^{2}, x_{1}^{3}\end{array}$ & $\mathcal{O}_{2,2}=\left\{1, x_{1}, x_{2}, x_{1}^{2}\right\}$ & $\begin{array}{c}x_{2}^{2}-x_{1}^{2} \\
x_{1} x_{2}-x_{1}^{2} \\
x_{1}^{2} x_{2}, x_{1}^{3}\end{array}$ \\
\hline none & - & $\mathcal{O}_{2.3}=\left\{1, x_{1}, x_{2}, x_{1} x_{2}\right\}$ & $\begin{array}{c}x_{1}^{2}-x_{1} x_{2}, \\
x_{2}^{2}-x_{1} x_{2}, \\
x_{1}^{2} x_{2}, x_{1} x_{2}^{2}\end{array}$ \\
\hline
\end{tabular}

TABLE 2. All admissible order ideals of $I_{2}=\left\langle x_{1}^{2}-x_{1} x_{2}, x_{2}^{2}-x_{1} x_{2}, \mathbb{T}_{=3}^{2}\right\rangle_{K[\mathbb{X}]}$ over the ring $K\left[x_{1}, x_{2}\right]$.

Clearly, as a vector space, every ideal $I$ has a degree filtration $I=\bigcup_{i \in \mathbb{N}} I^{\leq i}$ where $I^{\leq i}:=\{p \in$ $I \mid \operatorname{deg}(p) \leq i\}$. For a set of monomials $\mathcal{O}$ we define $\mathcal{O}^{=i}:=\{m \in \mathcal{O} \mid \operatorname{deg}(m)=i\}$, and similarly $\mathcal{O}^{\leq i}:=\{m \in \mathcal{O} \mid \operatorname{deg}(m) \leq i\}$. In the following we will also consider the special class of order ideals preserving the degree filtration, which are called degree-compatible:

Definition 2.4. Let $I \subseteq K[\mathbb{X}]$ be a zero-dimensional ideal and let $\mathcal{O} \subseteq \mathbb{T}^{n}$ be an order ideal. Then $\mathcal{O}$ is degree-compatible (to $I$ ) if

$$
\left|\mathcal{O}^{=i}\right|=\left|\mathbb{T}_{=i}^{n}\right|-\operatorname{dim} \frac{I \leq i}{I \leq i-1}
$$

for all $i \in \mathbb{N}$.

Thus, the $\mathcal{O}$-border basis of a zero-dimensional ideal $I$ with respect to any degree-compatible order ideal $\mathcal{O}$ has a pre-determined size for each degree $i \in \mathbb{N}$. Intuitively, the degree-compatible order ideals are those that correspond to degree-compatible orderings on the monomials. The important difference is that the orderings do not have to be term orderings. The definition above only requires local compatibility with multiplication if $\mathcal{O}$ is a degree-compatible order ideal and thus downwardly closed, i.e., if $p, q$ are polynomials and $\operatorname{deg}(p)<\operatorname{deg}(q)$ then $p \leq q$. Not all order ideals are degree-compatible as we will see in the following two examples.

Example 2.5 (Degree-compatible order ideals). For finding degree-compatible ideals, particularly suitable term orderings are the degree-compatible ones, like deglex and degrevlex, where $m_{1}>m_{2}$ for all monomials $m_{1}, m_{2}$ with $\operatorname{deg}\left(m_{1}\right)>\operatorname{deg}\left(m_{2}\right)$. Using the Gröbner basis $\mathcal{G}$ of an ideal $I$ under a degree-compatible term ordering, the low-degree parts $I^{\leq i}$ of $I$ can be easily determined using the elements of $\mathcal{G}$ of degree at most $i$, leading to the formula

$$
\operatorname{dim} \frac{I^{\leq i}}{I \leq i-1}=\left|\left\{m \in \mathbb{T}_{=i}^{n}: \exists g \in \mathcal{G}: \operatorname{LT}_{\sigma}(g) \mid m\right\}\right|
$$


from which (2.3) easily follows for the order ideal $\mathcal{O}$ coming from $\mathcal{G}$, i.e., admissible order ideals coming from a degree-compatible term ordering are degree-compatible.

Now it is easy to check that in Example 2.3, all the admissible order ideals are degree-compatible except $\mathcal{O}_{1,1}=\left\{1, x_{2}, x_{2}^{2}\right\}$ for $I_{1}=\left\langle x_{1} x_{2}-x_{2}-x_{1}+1, x_{2}^{2}+x_{1}-1\right\rangle_{K[\mathbb{X}]}$.

Example 2.6 (Generic ideal). Let $k$ and $n$ be positive integers and let $\left\{a_{i j}\right\}_{i \in[n], j \in[k]}$ be algebraically independent real numbers over $\mathbb{Q}$. Let $I$ be the ideal of polynomials in the variables $x_{1}, \ldots, x_{n}$ which are zero on the points $\left(a_{1 j}, \ldots, a_{n j}\right)$ for $j \in[k]$, i.e., it is the vanishing ideal of those points. Thus, the ideal is zero-dimensional, and $K[\mathbb{X}] / I$ has dimension $k$.

Every $k$ distinct monomials form a complementary basis of $I$, since they are linearly independent on the $k$ points $\left(a_{1 j}, \ldots, a_{n j}\right)$. An equivalent formulation of linear independence is that the determinant of the matrix formed by the values of the monomials on these points is non-zero. The determinant is indeed non-zero, as it is a non-trivial polynomial of the algebraic independent $a_{i j}$ with integer coefficients.

In particular, every order ideal of size $k$ is an order ideal of $I$. The degree-compatible order ideals are the ones where the monomials have the least possible degree, i.e., consisting of all monomials of degree less than $l$ and in addition $k-\left(\begin{array}{c}n+l-1 \\ l-1\end{array}\right)$ monomials of degree $l$, where $l$ is the smallest non-negative integer satisfying $k \leq\left(\begin{array}{c}n+l \\ l\end{array}\right)$, i.e., there are at least $k$ monomials of degree at most $l$.

2.2. Computing stable spans. Without proofs, we recall the underlying stable span computation of the border basis algorithm in [35] as it will serve as a basis for our algorithm. The interested reader is referred to [34,36] for a general introduction to border bases and to [35] in particular for an introduction to the border basis algorithm.

The border basis algorithm in [35] calculates border bases of zero-dimensional ideals with respect to an order ideal $\mathcal{O}$ which is induced by a degree-compatible term ordering $\sigma$ by successively generating a vector space approximation of the ideal. These approximations are generated via the following vector space neighborhood extensions:

Definition 2.7. (cf. [35, Definition 7.1 and the paragraph preceding Proposition 13]) Let $V \subseteq K[\mathbb{X}$ ] be a vector space. We define the neighborhood extension of $V$ to be

$$
V^{+}:=V+V x_{1}+\cdots+V x_{n} .
$$

For a finite set $W$ of polynomials, its neighborhood extension is

$$
W^{+}=W \cup W x_{1} \cup \cdots \cup W x_{n} .
$$

Note that for a given set of polynomials $W$ such that $\langle W\rangle_{K}=V$ we have $\left\langle W^{+}\right\rangle_{K}=\langle W\rangle_{K}^{+}=V^{+}$ as multiplication with $x_{i}$ is a $K$-linear map. It thus suffices to perform the neighborhood extension on a set of generators $W$ of $V$.

Let $F$ be a finite set of polynomials and let $L \subseteq \mathbb{T}^{n}$ be an order ideal, representing our computational universe. We would like to compute the ideal generated by $F$ restricted to our universe, i.e., $\langle F\rangle_{K[\mathbb{X}]} \cap\langle L\rangle_{K}$. We are mainly concerned with finite sets $L \subseteq \mathbb{T}^{n}$.

Note that $F \cap\langle L\rangle_{K}=\{f \in F \mid \operatorname{supp}(f) \subseteq L\}$, i.e., $F \cap\langle L\rangle_{K}$ contains only those polynomials that lie in the vector space generated by $L$. Clearly, $\langle F\rangle_{K} \cap\left\langle\mathbb{T}_{\leq d}^{n}\right\rangle_{K}=\langle F\rangle_{K}^{\leq d}$. Using neighborhood extension we define:

Definition 2.8. (Cf. [35, Definition 10]) Let $L$ be an order ideal and let $F$ be a finite set of polynomials such that $\operatorname{supp}(F) \subseteq L$. The set $F$ is L-stabilized if $\left\langle F^{+}\right\rangle_{K} \cap\langle L\rangle_{K}=\langle F\rangle_{K}$. The L-stable span $F_{L}$ of $F$ is the smallest vector space $V$ containing $F$ satisfying $V^{+} \cap\langle L\rangle_{K}=V$.

The basic example of an $L$-stabilized set is a set of generators for the intersection $I \cap L$ of an ideal $I$ with $L$, but not all $L$-stabilized sets have necessary this form. For example, $L$ itself is $L$-stabilized 
for any order ideal $L$. For $L=\left\{1, x_{1}, x_{2}, x_{1}^{2} x_{2}^{2}, x_{2}^{3}\right\}$, the set $\left\{x_{1}+x_{2}, x_{1}^{2}, x_{2}^{2}, x_{2}^{3}\right\}$ is $L$-stabilized, but $\left\{x_{1}+x_{2}, x_{1}^{2}\right\}$ is not.

The following simple observation will be helpful later.

Remark 2.9. The $L$-stable span of a finite set $F$ depends only on the vector space $\langle F\rangle_{K}$ spanned by $F$, as $\left\langle F^{+}\right\rangle_{K}=\langle F\rangle_{K}^{+}$.

A straightforward construction of the $L$-stable span of $F$ is to inductively define the following increasing sequence of vector spaces:

$$
F_{0}:=\langle F\rangle_{K} \quad \text { and } \quad F_{k+1}:=F_{k}^{+} \cap\langle L\rangle_{K} \text { for } k>0 .
$$

The union $\bigcup_{k \geq 0} F_{k}$ is the $L$-stable span $F_{L}$ of $F$.

In the following we will explain how the $L$-stable span can be computed explicitly for $L=\mathbb{T}_{<d}^{n}$. We will use a modified version of Gaussian elimination as a tool, which allows us to extend a given basis $V$ with a set $W$ as described in the following:

Lemma 2.10. [35, Lemma 12] Let $V=\left\{v_{1}, \ldots, v_{r}\right\} \subseteq K[\mathbb{X}] \backslash\{0\}$ be a finite set of polynomials such that $\operatorname{LT}\left(v_{i}\right) \neq \operatorname{LT}\left(v_{j}\right)$ whenever $i, j \in[r]$ with $i \neq j$ and $\operatorname{LC}\left(v_{i}\right)=1$ for all $i \in[r]$. Further let $G$ be a finite set of polynomials. Then Algorithm 2.11 computes a finite set of polynomials $W \subseteq K[\mathbb{X}]$ with

(1) $\mathrm{LC}(w)=1$ for all $w \in W$,

(2) $\operatorname{LT}\left(u_{1}\right) \neq \operatorname{LT}\left(u_{2}\right)$ for any distinct $u_{1}, u_{2} \in V \cup W$, and

(3) $\langle V \cup W\rangle_{K}=\langle V \cup G\rangle_{K}$.

$(V, W$ may be empty.)

Algorithm 2.11 (Gaussian Elimination for polynomials-GaussE1).

Input: $V, G$ finite set of polynomials, $0 \notin V$ (as in Lemma 2.10).

Output: $W \subseteq K[\mathbb{X}]$ finite set of polynomials (as in Lemma 2.10).

(1) Let $H:=G$ and $\eta:=0$.

(2) If $H=\varnothing$ then return $W:=\left\{v_{r+1}, \ldots, v_{r+\eta}\right\}$ and stop.

(3) Choose $f \in H$ and remove it from $H$. Let $i:=1$.

(4) If $f=0$ then go to step (2)

(5) If $i>r+\eta$ then put $\eta:=\eta+1$ and let $v_{r+\eta}:=f / \operatorname{LC}(f)$. Go to step (2)

(6) If $\operatorname{LT}(f)=\operatorname{LT}\left(v_{i}\right)$ then replace $f$ with $f-\operatorname{LC}(f) \cdot v_{i}$. Set $i:=1$ and go to step (5)

(7) Set $i:=i+1$. Go to step (5)

We can now compute the $L$-stable span using the Gaussian elimination algorithm 2.11

Lemma 2.12. [35, Proposition 13] Let $L=\mathbb{T}_{\leq d}^{n}$ and $F \subseteq K[\mathbb{X}]$ be a finite set of polynomials supported on $L$. Then Algorithm 2.13 computes a vector space basis $V$ of $F_{L}$ with pairwise different leading terms.

Algorithm 2.13 ( $L$-stable span computation-LStabSpan).

Input: $F$, L as in Lemma 2.12 .

Output: $V$ as in Lemma2.12.

(1) $V:=$ GaussEl $(\varnothing, F)$.

(2) $W^{\prime}:=$ Gausse1 $\left(V, V^{+} \backslash V\right)$.

(3) $W:=\left\{w \in W^{\prime} \mid \operatorname{supp}(w) \subseteq L\right\}=\left\{w \in W^{\prime} \mid \operatorname{deg}(w) \leq d\right\}$.

(4) If $W \neq \varnothing$ set $V:=V \cup W$ and go to step (2)

(5) Return $V$.

The rationale for computing a stable span approximation is due to the following proposition that serves as a criterion for testing whether an order ideal $\mathcal{O}$ supports a border basis. 
Proposition 2.14. [35, Proposition 16] Let L be an order ideal. Further let Ĩ be an L-stabilized generating vector subspace of a zero-dimensional ideal $I \subseteq K[\mathbb{X}]$, i.e., $\tilde{I}^{+} \cap\langle L\rangle_{K}=\tilde{I}$ and $\langle\tilde{I}\rangle_{K[\mathbb{X}]}=I$. If $\mathcal{O}$ is an order ideal such that $\langle L\rangle_{K}=\tilde{I} \oplus\langle\mathcal{O}\rangle_{K}$ and $\partial \mathcal{O} \subseteq L$ then $\mathcal{O}$ supports a border basis of $I$.

We obtain the following corollary, which will be helpful later.

Corollary 2.15. Let $\tilde{I}$ be an $\mathbb{T}_{\leq d}^{n}$-stabilized vector space satisfying $\tilde{I}+\left\langle\mathbb{T}_{\leq d-1}^{n}\right\rangle_{K}=\left\langle\mathbb{T}_{\leq d}^{n}\right\rangle_{K}$. Then $\langle\tilde{I}\rangle_{K[\mathbb{X}]} \cap\left\langle\mathbb{T}_{\leq d}^{n}\right\rangle_{K}=\tilde{I}$ and $K[\mathbb{X}] /\langle\tilde{I}\rangle_{K[\mathbb{X}]} \cong\left\langle\mathbb{T}_{\leq d}^{n}\right\rangle_{K} / \tilde{I}$

Proof. We apply Proposition 2.14 with the choice $L:=\mathbb{T}_{\leq d^{\prime}}^{n} I:=\langle\tilde{I}\rangle_{K[\mathbb{X}]}$ and $\mathcal{O}:=\mathbb{T}_{\leq d}^{n} \backslash \operatorname{LT}(\tilde{I})$ where the leading terms are with respect to any degree-compatible ordering (i.e., $m_{1}<m_{2}$ whenever deg $\left.m_{1}<\operatorname{deg} m_{2}\right)$. Clearly, $\left\langle\mathbb{T}_{\leq d}^{n}\right\rangle_{K}=\tilde{I} \oplus\langle\mathcal{O}\rangle_{K}$. The condition $\tilde{I}+\left\langle\mathbb{T}_{\leq d-1}^{n}\right\rangle_{K}=\left\langle\mathbb{T}_{\leq d}^{n}\right\rangle_{K}$ ensures that $\mathcal{O}$ consists of monomials of degree less than $d$, so $\partial \mathcal{O} \subseteq \mathbb{T}_{\leq d}^{n}$. Hence the proposition applies, and we obtain $K[\mathbb{X}]=I \oplus\langle\mathcal{O}\rangle_{K}$. Together with $\left\langle\mathbb{T}_{\leq d}^{n}\right\rangle_{K}=\tilde{I} \oplus\langle\mathcal{O}\rangle_{K}$ this gives $I \cap\left\langle\mathbb{T}_{\leq d}^{n}\right\rangle_{K}=\tilde{I}$, and $K[\mathbb{X}] /\langle\tilde{I}\rangle_{K[\mathbb{X}]} \cong\langle\mathcal{O}\rangle_{K} \cong\left\langle\mathbb{T}_{\leq d}^{n}\right\rangle_{K} / \tilde{I}$.

For a worst-case upper bound on $d$, we will use the dimension of $K[\mathbb{X}] / I$. The necessary technical background is the following lemma.

Lemma 2.16. Let I be a zero-dimensional ideal of $K[\mathbb{X}]$, and let $d:=\operatorname{dim} K[\mathbb{X}] / I$. Then

(1) $I \leq d / I \leq d-1 \cong\left\langle\mathbb{T}_{=d}^{n}\right\rangle_{K}$ and

(2) $\left\langle\mathbb{T}_{\leq d-1}^{n}\right\rangle_{K} / I \leq d-1 \cong K[\mathbb{X}] / I$.

Proof. Choose a degree-compatible term ordering. The associated order ideal (as every order ideal of size $d$ ) contains monomials of degree less than $d$. This proves $I+\left\langle\mathbb{T}_{\leq d-1}^{n}\right\rangle_{K}=K[\mathbb{X}]$, from which the statements easily follow via the modular law $((A+B) \cap C=A+(B \cap C)$ for all subspaces with $A \subseteq C)$.

\section{THE ORDER IDEAL POLYTOPE}

We will now introduce the order ideal polytope $P(I)$ that characterizes all admissible order ideals, i.e., order ideals supporting a border basis for a given zero-dimensional ideal $I$, in an abstract fashion completely independent of the stable span approximation. Its role will be crucial for the later computation of border bases for general order ideals. We will first focus on its properties and structure, then in Section 3.2, we will consider the computational aspect. We will show that the integral points of the order ideal polytope $P(I)$ are in bijection with the admissible order ideals of a given zero-dimensional ideal $I$. In order to do so, we approach the problem from a polyhedral point of view to capture the intrinsic combinatorics for the admissibility condition $K[\mathbb{X}]=I \oplus\langle\mathcal{O}\rangle_{K}$ on the one hand and $\mathcal{O}$ being an order ideal on the other hand. The role of the polyhedral description becomes prominent in Section 3.2 when the directness of the sum $I \oplus\langle\mathcal{O}\rangle_{K}$ is rephrased in the language of matrices and vector space bases.

3.1. Theoretical point of view. We start with defining the order ideal polytope whose integral solutions are exactly the characteristic vectors of order ideals admissible for a fixed zero dimensional ideal $I$. As we will see, the defining inequalities express various properties of admissible order ideals. 
Definition 3.1. Let $I$ be a zero-dimensional ideal. Its order ideal polytope $P(I)$ is defined by the following system of inequalities with variables $z_{m}$ for $m \in \mathbb{T}_{\leq d-1}^{n}$, where $d:=\operatorname{dim} K[\mathbb{X}] / I$.

$$
\begin{aligned}
z_{m_{1}} & \geq z_{m_{2}} & \forall m_{1}, m_{2} \in \mathbb{T}_{\leq d-1}^{n}: m_{1} \mid m_{2} \\
\sum_{m \in \mathbb{T}_{\leq d-1}^{n}} z_{m} & =d & \\
\sum_{m \in U} z_{m} & \leq \operatorname{dim}\langle U \cup I\rangle_{K} / I & \forall U \subseteq \mathbb{T}_{\leq d-1}^{n}:|U|=d \\
z_{m} & \in[0,1] & \forall m \in \mathbb{T}_{\leq d-1}^{n} .
\end{aligned}
$$

To obtain a finite dimensional polytope, we bounded the degree of the monomials by $\operatorname{dim} K[\mathbb{X}] / I$ from above. This bound is large enough to contain all occurring monomials as we will see below. In a first step we relate the order ideal polytope with admissible order ideals. Recall that $\Lambda(I)$ denotes the set of admissible order ideals, i.e., order ideals supporting a border basis of a zero-dimensional ideal $I$.

Theorem 3.2. Let I be a zero-dimensional ideal. There is an explicit bijection $\xi$ between the set $\Lambda(I)$ of admissible order ideals of I and the set of integral points of the order ideal polytope $P(I)$ of I. The bijection is given by

$$
\begin{gathered}
\xi: P(I) \cap\{0,1\}^{\mathbb{T}_{\leq d-1}^{n} \rightarrow \Lambda(I)} \\
\xi(z)=\mathcal{O}(z):=\left\{m \in \mathbb{T}_{\leq d-1}^{n} \mid z_{m}=1\right\} .
\end{gathered}
$$

Proof. We show that the domain $P(I) \cap\{0,1\}^{\mathbb{T}_{\leq d-1}^{n}}$ of $\xi$ is exactly the set of characteristic vectors of all order ideals $\mathcal{O} \subseteq \mathbb{T}_{\leq d-1}^{n}$ admissible for $I$. It will immediately follow that $\xi$ is a well-defined bijection onto $\Lambda(I)^{\leq d-1}$, the set of admissible order ideals of $I$ with all monomials having degree less than $d$. Actually, this is the set of all admissible order ideals of $I$, as every admissible order ideal $\mathcal{O}$ of $I$ has size the dimension $d$ of the factor $K[\mathbb{X}] / I$, and hence can only contain monomials up to degree $d-1$.

Let $z \in\{0,1\}^{\mathbb{T}_{\leq d-1}^{n}}$ be a $0 / 1$ vector. It is the characteristic vector of the set $\mathcal{O}(z):=\left\{m \in \mathbb{T}_{\leq d-1}^{n} \mid\right.$ $\left.z_{m}=1\right\}$. Recall that the set $\mathcal{O}(z)$ is an admissible order ideal of $I$ if and only if the following hold:

(1) $\mathcal{O}(z)$ is an order ideal, i.e., $m_{2} \in \mathcal{O}(z)$ implies $m_{1} \in \mathcal{O}(z)$ for all monomials $m_{1}$ and $m_{2}$ with $m_{1} \mid m_{2}$.

(2) $|\mathcal{O}(z)|=d$.

(3) The image of $\mathcal{O}(z)$ in $K[\mathbb{X}] / I$ is linearly independent.

The last two conditions together are clearly an equivalent formulation of $K[\mathbb{X}]=\langle\mathcal{O}(z)\rangle_{K} \oplus I$, using that $\mathcal{O}(z)$ is a set of linearly independent elements in $K[\mathbb{X}]$.

Now we rewrite these conditions for the characteristic vector $z$. Condition (1) for fixed monomials $m_{1} \mid m_{2}$ is obviously equivalent to $z_{m_{1}} \geq z_{m_{2}}$. Therefore Condition (1) is equivalent to (3.1a). Similarly, as $\sum_{m \in \mathbb{T}_{<d-1}^{n}} z_{m}=|\mathcal{O}(z)|$, Condition (2) is equivalent to (3.1b).

As of Condition (3), we first give a more complex but equivalent formulation:

$$
|U \cap \mathcal{O}(z)| \leq \operatorname{dim}\langle U \cup I\rangle_{K} / I, \quad \text { for all } U \subseteq \mathbb{T}_{\leq d-1}^{n} \text { with }|U|=d .
$$

i.e., the size of $U \cap \mathcal{O}(z)$ is at most the dimension of the vector space generated by the image of $U$ in the factor $K[\mathbb{X}] / I$. This is obviously necessary for the image of $\mathcal{O}(z)$ to be linearly independent in the factor, as then the image of $U \cap \mathcal{O}(z)$ is independent, and contained in $\langle U \cup I\rangle_{K} / I$. (For necessity, the size of $U$ does not matter.) For sufficiency choose $U:=\mathcal{O}(z)$, showing that the image of $\mathcal{O}(z)$ spans a subspace of $K[\mathbb{X}] /$ I of size at least that of $\mathcal{O}(z)$, i.e., that the image of $\mathcal{O}(z)$ is linearly independent. Thus Condition (3) is equivalent to (3.2), which is (3.1c) using $|U \cap \mathcal{O}(z)|=\sum_{m \in U} z_{m}$. 
All in all, a $0 / 1$ vector $z \in\{0,1\}^{\mathbb{T}_{\leq d-1}^{n}}$ is the characteristic vector of an order ideal admissible for $I$ if and only if it satisfies (3.1a), (3.1b) and (3.1c). In other words, $P(I) \cap\{0,1\}^{\mathbb{T}_{\leq d-1}^{n}}$ is the set of characteristic vectors of order ideals $\mathcal{O} \subseteq \mathbb{T}_{\leq d-1}^{n}$ admissible to $I$, as claimed. (The remaining inequalities (3.1d) of $P(I)$ are satisfied by all $0 / 1$ vectors.)

Example 3.3 (Order ideal polytope). As an easy example we determine the order ideal polytope $P\left(I_{2}\right)$ of the ideal $I_{2}=\left\langle x_{1}^{2}-x_{1} x_{2}, x_{2}^{2}-x_{1} x_{2}, \mathbb{T}_{=3}^{2}\right\rangle_{K[\mathbb{X}]}$ from Example 2.3, First we derive several valid inequalities for $P\left(I_{2}\right)$ in order to obtain a simple description.

Recall that $K\left[x_{1}, x_{2}\right] / I_{2}$ has dimension $d=4$, so the coordinates of the polytope are indexed by monomials up to degree 3 . As the ideal $I$ contains $\mathbb{T}_{=3}^{2}$, by (3.1c) applied to $U=\mathbb{T}_{=3}^{2}$, which consists of exactly 4 monomials,

$$
\sum_{m \in \mathbb{T}_{=3}^{2}} z_{m} \leq 0
$$

Together with $z_{m} \geq 0$ for all $m$, this implies

$$
z_{m}=0, \quad \text { whenever } \operatorname{deg} m=3 .
$$

Hence from now on we can omit variables indexed by degree-three monomials as they are 0 .

Now we apply (3.1c) again, but this time for $U=\mathbb{T}_{=2}^{2} \cup\left\{x_{1}^{3}\right\}$ (the role of the monomial $x_{1}^{3}$ is simply to pad $U$ ensuring that $U$ has 4 elements) and derive

$$
z_{x_{1}^{2}}+z_{x_{1} x_{2}}+z_{x_{2}^{2}} \leq 1 \text {. }
$$

Together with

$$
z_{1}+z_{x_{1}}+z_{x_{2}}+z_{x_{1}^{2}}+z_{x_{1} x_{2}}+z_{x_{2}^{2}}=4
$$

by (3.1b) and $z_{m} \leq 1$ for all $m \in \mathbb{T}_{\leq 1}^{2}$ by (3.1d), we obtain

$$
\begin{gathered}
z_{1}=z_{x_{1}}=z_{x_{2}}=1, \\
z_{x_{1}^{2}}+z_{x_{1} x_{2}}+z_{x_{2}^{2}}=1 .
\end{gathered}
$$

All in all, the polytope $P\left(I_{2}\right)$ satisfies the following inequalities:

$$
\begin{aligned}
& z_{m}=0, \quad \text { whenever } \operatorname{deg} m=3, \\
& z_{1}=z_{x_{1}}=z_{x_{2}}=1 \text {, } \\
& z_{x_{1}^{2}}+z_{x_{1} x_{2}}+z_{x_{2}^{2}}=1 \text {, } \\
& z_{x_{1}^{2}}, z_{x_{1} x_{2}}, z_{x_{2}^{2}} \geq 0 \text {. }
\end{aligned}
$$

This system defines a triangle with the following vertices and gives rise to the order ideals $\mathcal{O}_{2,2}=$ $\left\{1, x_{1}, x_{2}, x_{1}^{2}\right\}, \mathcal{O}_{2,3}=\left\{1, x_{1}, x_{2}, x_{1} x_{2}\right\}$, and $\mathcal{O}_{2,1}=\left\{1, x_{1}, x_{2}, x_{2}^{2}\right\}$ :

- $\left(z_{x_{1}^{2}}=1, z_{x_{1} x_{2}}=0, z_{x_{2}^{2}}=0\right)$

[characteristic vector of $\mathcal{O}_{2,2}$ ]

- $\left(z_{x_{1}^{2}}=0, z_{x_{1} x_{2}}=1, z_{x_{2}^{2}}=0\right)$

[characteristic vector of $\mathcal{O}_{2,3}$ ]

- $\left(z_{x_{1}^{2}}=0, z_{x_{1} x_{2}}=0, z_{x_{2}^{2}}=1\right)$

[characteristic vector of $\mathcal{O}_{2,1}$ ]

We conclude that the $P\left(I_{2}\right)$ is the triangle with vertices the characteristic vectors of the admissible order ideals of $I_{2}$, listed in Table 2 .

Example 3.4 (A non-integral order ideal polytope). In contrast to $P\left(I_{2}\right)$ of the previous example, the order ideal polytope $P\left(I_{1}\right)$ of $I_{1}$ from Example 2.3 is not the convex hull of the characteristic vectors of the admissible order ideals of $I_{1}$ but a proper relaxation, i.e., $P\left(I_{1}\right)$ is more than the line segment of $\mathcal{O}_{1,1}$ and $\mathcal{O}_{1,2}$. A point of $P\left(I_{1}\right)$ lying outside this segment is the one with all its coordinates being $1 / 2$ :

$$
z_{1}=z_{x_{1}}=z_{x_{2}}=z_{x_{1}^{2}}=z_{x_{1} x_{2}}=z_{x_{2}^{2}}=1 / 2
$$




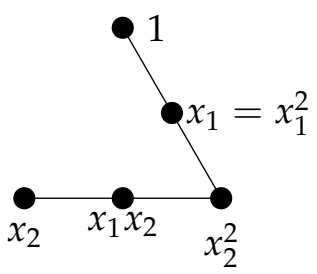

FIGURE 3.1. Linear dependence relations between low-degree monomials in the factor $K\left[x_{1}, x_{2}\right] / I_{1}$ of dimension 3 . The monomials are depicted in the projective space of the factor, to save a dimension.

Recall that $K\left[x_{1}, x_{2}\right] / I_{1}$ has dimension 3.

This point satisfies the system (3.1), from which only (3.1c) requires explanation. For computing the right-hand side of (3.1c), the key is to determine the linear dependence relations between the monomials of degree at most 2 in the factor space $K\left[x_{1}, x_{2}\right] / I_{1}$. These can be easily read off from Figure 3.1 depicting the projective space of the factor $K\left[x_{1}, x_{2}\right] / I_{1}$, which has dimension 2 . To verify the figure, note that $\mathcal{O}_{1,1}=\left\{1, x_{2}, x_{2}^{2}\right\}$ is a basis of the factor, and hence forms a triangle in the projective space. As $x_{1}+x_{2}^{2}-1, x_{1}^{2}-x_{1}, x_{1} x_{2}+x_{2}^{2}-x_{2}$ are all elements of $I_{1}$, we immediately see that $x_{1} x_{2}$ is a third point on the line joining $x_{2}$ and $x_{2}^{2}$, and $x_{1}$ is a third point on the line of 1 and $x_{2}^{2}$, while $x_{1}^{2}$ is the same point as $x_{1}$.

Now from Figure 3.1 it is immediate that any subset $U \subseteq \mathbb{T}_{\leq 2}^{2}$ of size 3 has dimension at least 2 in $K\left[x_{1}, x_{2}\right] / I_{1}$. Therefore the right-hand side of (3.1c), is at least 2, while the left-hand side is exactly $3 / 2$, and therefore the inequality holds as claimed.

Remark 3.5. Adding to (3.1) the equality $z_{1}=1$ and requiring (3.1c) for all $U \subseteq \mathbb{T}_{<d-1}^{n}$ would still be insufficient to describe the convex hull of admissible order polytopes of $I_{1}$ : the following point still lies outside the convex hull while satisfying even the additional constraints:

$$
z_{0}=1, \quad z_{x_{1}}=z_{x_{2}}=z_{x_{1}^{2}}=z_{x_{1} x_{2}}=z_{x_{2}^{2}}=2 / 5 .
$$

3.2. Computational point of view. From a computational perspective, the system (3.1) defining the order ideal polytope contains dimensions $\operatorname{dim}\langle U \cup I\rangle_{K} / I$, which are computationally challenging to determine. Therefore in this subsection we provide a modified description of the order ideal polytope, well suited for computations.

Let $M \subseteq K[\mathbb{X}]$ be a finite set of polynomials, and let $M^{=i}$ denote the set of polynomials in $M$ with total degree $i$. We would like to have $M$ to be a vector space basis of $I \leq d$ reflecting the degree filtration of $I$. The following definition will be helpful.

Definition 3.6. Let $M$ be a finite set of non-zero polynomials of degree at most $\ell$ for some $\ell \in \mathbb{N}$. Then $M$ is in canonical form if the leading term of any element of $M$ does not occur in the other elements.

Here the ordering on monomials can be any degree-compatible (total) ordering (i.e., $m_{1}<m_{2}$ for all monomials $m_{1}, m_{2}$ with $\operatorname{deg} m_{1}<\operatorname{deg} m_{2}$ ), and need not be a term ordering. Clearly, any vector space basis can be brought into canonical form via Gaussian elimination. The coefficient matrix $A \in K^{M \times \mathbb{T}_{\leq \ell}^{n}}$ of $M$ is the matrix where the rows are indexed by the elements of $M$, and the columns are indexed by the monomials of degree at most $\ell$, and the entries are the coefficients of the monomials in the elements of $M$. In other words, $A_{f, m}=a_{m}$ for $f=\sum_{m \in \mathbb{T}_{\leq \ell}^{n}} a_{m} m \in M$. A visual interpretation of a set $M$ in canonical form can be found in Figure 3.2 using the coefficient matrix.

The following lemma summarizes the required properties of a generating set $M$ of an ideal $I$ sufficient to describe the degree filtration of $I \leq d$. 


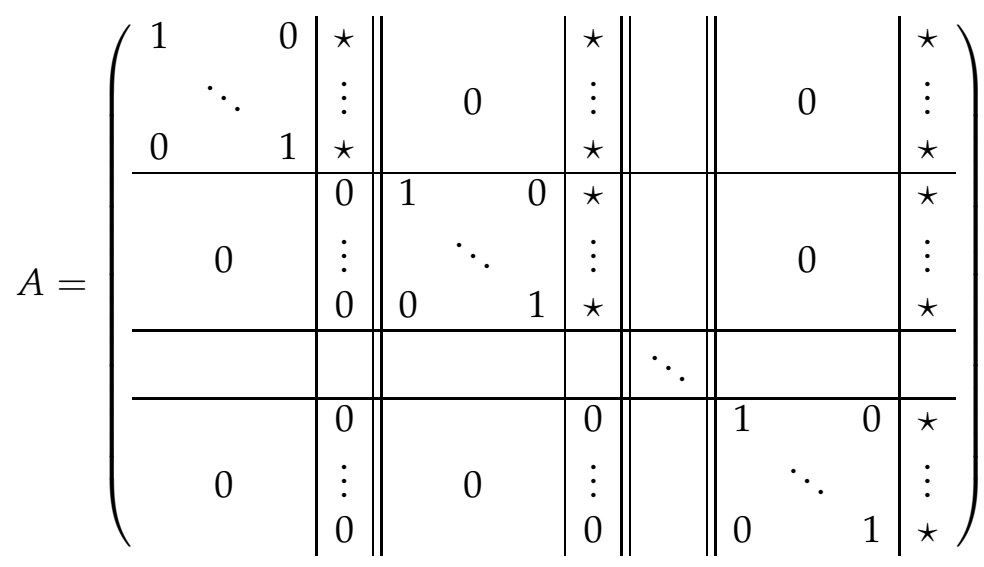

FIgURE 3.2. Coefficient matrix of a set of polynomials in canonical form. Double lines separate samedegree blocks of monomials.

Lemma 3.7. Let $M$ be in canonical form and $\mathbb{T}_{\leq d}^{n}$-stabilized. Further assume $\mathbb{T}_{=d}^{n} \subseteq\langle M\rangle_{K}+\left\langle\mathbb{T}_{\leq d-1}^{n}\right\rangle_{K}$. Then the following hold for all $i \in[d]$ :

(1) A basis for $\langle M\rangle_{K[\mathbb{X}]}^{\leq i} /\langle M\rangle_{K[\mathbb{X}]}^{\leq i-1}$ is the image of $M^{=i}$.

(2) $\langle M\rangle_{K[\mathbb{X}]}^{\leq i}=\left\langle\bigcup_{j \leq i} M^{=j}\right\rangle_{K}$

(3) $\left\langle M^{=i}\right\rangle_{K}^{<i}=0$ and thus $\left\langle M^{=i}\right\rangle_{K}^{<i} \subseteq\left\langle\bigcup_{0 \leq j \leq i-1} M^{=j}\right\rangle_{K}$

Proof. We first show that $\left\langle M^{=i}\right\rangle_{K}^{<i}=0$ for all $i \in[d]$. Let $i \in[d]$ be arbitrary and observe that each nonzero element $p \in M^{=i}$ has degree $i$. As $M$ is in canonical form, the polynomials in $M^{=i}$ are interreduced (see the matrix in Figure 3.2 for Definition 3.6) and thus each nonzero element $p \in\left\langle M^{=i}\right\rangle_{K}$ has degree $i$.

By Corollary 2.15, we have $\langle M\rangle_{K[\mathbb{X}]} \cap\langle L\rangle_{K}=\langle M\rangle_{K}$. Hence $\langle M\rangle_{K[\mathbb{X}]}^{\leq i}=\langle M\rangle_{K}^{\leq i}$ for $i \in[d]$. Now the statements of the lemma are obvious consequences of $M$ being in canonical form.

The following lemma provides us a practical way to compute the sizes of the degree components of degree-compatible order ideals, which are the same for all order ideals of a given ideal.

Lemma 3.8. Let $M$ be in canonical form and $\mathbb{T}_{\leq d}^{n}$-stabilized. Further let $\mathcal{O}$ be an order ideal of $\langle M\rangle_{K[\mathbb{X}]^{\prime}}$ and $d=\max _{m \in \partial \mathcal{O}} \operatorname{deg}(m)$. Let us assume $\mathbb{T}_{=d}^{n} \subseteq\langle M\rangle_{K}+\mathbb{T}_{\leq d-1}^{n}$. Then $\mathcal{O}$ is degree-compatible if and only if

$$
\left|\mathcal{O}^{=i}\right|=\left|\mathbb{T}_{=i}^{n}\right|-\left|M^{=i}\right|
$$

for every $i \in[d]$.

Proof. In view of Definition 2.4 it suffices to observe that $I^{\leq i} / I^{\leq i-1}$ has the image of $M^{=i}$ as a basis by Lemma 3.7(1).

We are ready to provide a reformulation of the definition of order ideal polytopes, which is better suited for actual computations, partly as it involves only direct matrix operations via replacing dimensions with ranks of submatrices. While $d$ will still be the dimension of $K[\mathbb{X}] /\langle M\rangle_{K[\mathbb{X}]}$, we do not require explicit a priori knowledge, but rather formulate alternative, sufficient conditions, which are easier to verify by an algorithm. 
Lemma 3.9. Let $M$ be $\mathbb{T}_{\leq d}^{n}$-stabilized and in canonical form for some $d \in \mathbb{N}$. Suppose $\langle M\rangle_{\bar{K}}^{\leq d} /\langle M\rangle_{\bar{K}}^{\leq d-1} \cong$ $\left\langle\mathbb{T}_{=d}^{n}\right\rangle_{K}$ and $d=\left|\mathbb{T}_{\leq d}^{n}\right|-|M|$. Then an alternative description of the order ideal polytope $P\left(\langle M\rangle_{K[\mathbb{X}]}\right)$ of $\langle M\rangle_{K[\mathbb{X}]}$ is given by the system of inequalities

$$
\begin{aligned}
z_{m_{1}} & \geq z_{m_{2}} & \forall m_{1}, m_{2} \in \mathbb{T}_{\leq d-1}^{n}: m_{1} \mid m_{2} \\
\sum_{m \in \mathbb{T}_{\leq d-1}^{n}} z_{m} & =d & \\
\sum_{m \in U} z_{m} & \geq|U|-\operatorname{rk}(\tilde{U}) & \forall U \subseteq \mathbb{T}_{\leq d-1}^{n}:|U|=\left|M^{\leq d-1}\right| \\
0 \leq z_{m} & \leq 1 & \forall m \in \mathbb{T}_{\leq d-1}^{n} .
\end{aligned}
$$

In (3.6c), the matrix $\tilde{U}$ is the submatrix of the coefficient matrix of $M \leq d-1$ consisting of only the columns indexed by monomials in $U$.

Proof. Let $I:=\langle M\rangle_{K[\mathbb{X}]}$. As $\mathbb{T}_{\leq d}^{n}=\langle M\rangle_{K}+\mathbb{T}_{\leq d-1}^{n}$ by assumption, Lemma 3.7provides $\langle M\rangle_{K[\mathbb{X}]} \cap$ $\left\langle\mathbb{T}_{\leq d}^{n}\right\rangle_{K}=\langle M\rangle_{K}$. Moreover, $K[\mathbb{X}]=\langle M\rangle_{K[\mathbb{X}]}+\mathbb{T}_{\leq d-1}^{n}$, hence via an application of the modular law $\left(\langle M\rangle_{K[\mathbb{X}]}+\mathbb{T}_{\leq d-1}^{n}\right) /\langle M\rangle_{K[\mathbb{X}]}=\mathbb{T}_{\leq d-1}^{n} /\left(\langle M\rangle_{K[\mathbb{X}]} \cap \mathbb{T}_{\leq d-1}^{n}\right)$, we obtain $K[\mathbb{X}] /\langle M\rangle_{K[\mathbb{X}]}=$ $\mathbb{T}_{\leq d-1}^{n} /\left\langle M^{\leq d-1}\right\rangle_{K}$. In particular, $\operatorname{dim} K[\mathbb{X}] /\langle M\rangle_{K[\mathbb{X}]}=\left|\mathbb{T}_{\leq d-1}^{n}\right|-\left|M^{\leq d-1}\right|=d$

As $d=\operatorname{dim} K[\mathbb{X}] /\langle M\rangle_{K[\mathbb{X}]}$, the only difference between the systems (3.1) and (3.6) is that (3.1C) is replaced by (3.6c). So we will show their equivalence modulo the other inequalities.

We start with (3.6C) for a fixed $U \subseteq \mathbb{T}_{\leq d-1}^{n}$, and make equivalent transformations to it. (For the following argument the size of $U$ is irrelevant.) Taking the difference with the equality (3.6b), we obtain

$$
\sum_{m \in \mathbb{T}_{\leq d-1}^{n} \backslash U} z_{m} \leq d-|U|+\operatorname{rk}(\tilde{U})
$$

Next we rewrite the right-hand side. Recall that $\tilde{U}$ is the submatrix obtained by restricting to the columns corresponding to the monomials in $U$, i.e., the coefficient matrix of the image of $M^{\leq d-1}$ in the factor $\mathbb{T}_{\leq d-1}^{n} /\left\langle\mathbb{T}_{\leq d-1}^{n} \backslash U\right\rangle_{K}$. Therefore

$$
\operatorname{rk}(\tilde{U})=\operatorname{dim} \frac{\left\langle M^{\leq d-1} \cup\left(\mathbb{T}_{\leq d-1}^{n} \backslash U\right)\right\rangle_{K}}{\left\langle\mathbb{T}_{\leq d-1}^{n} \backslash U\right\rangle_{K}}=\operatorname{dim}\left\langle M^{\leq d-1} \cup\left(\mathbb{T}_{\leq d-1}^{n} \backslash U\right)\right\rangle_{K}-\left|\mathbb{T}_{\leq d-1}^{n} \backslash U\right| .
$$

Thus the right-hand side of (3.7) becomes

$$
\begin{aligned}
d-|U|+\operatorname{rk}(\tilde{U}) & =d-|U|+\operatorname{dim}\left\langle M^{\leq d-1} \cup\left(\mathbb{T}_{\leq d-1}^{n} \backslash U\right)\right\rangle_{K}-\left|\mathbb{T}_{\leq d-1}^{n} \backslash U\right| \\
& =d-\left|\mathbb{T}_{\leq d-1}^{n}\right|+\operatorname{dim}\left\langle M^{\leq d-1} \cup\left(\mathbb{T}_{\leq d-1}^{n} \backslash U\right)\right\rangle_{K} \\
& =-\operatorname{dim}\left\langle M^{\leq d-1}\right\rangle_{K}+\operatorname{dim}\left\langle M^{\leq d-1} \cup\left(\mathbb{T}_{\leq d-1}^{n} \backslash U\right)\right\rangle_{K} \\
& =\operatorname{dim} \frac{\left\langle M^{\leq d-1} \cup\left(\mathbb{T}_{\leq d-1}^{n} \backslash U\right)\right\rangle_{K}}{\left\langle M^{\leq d-1}\right\rangle_{K}}=\operatorname{dim} \frac{\left\langle I \cup\left(\mathbb{T}_{\leq d-1}^{n} \backslash U\right)\right\rangle_{K}}{I},
\end{aligned}
$$


where the last equality follows via the modular law

$$
\frac{\left\langle M^{\leq d-1} \cup\left(\mathbb{T}_{\leq d-1}^{n} \backslash U\right)\right\rangle_{K}+I}{I}=\frac{\left\langle M^{\leq d-1} \cup\left(\mathbb{T}_{\leq d-1}^{n} \backslash U\right)\right\rangle_{K}}{\left\langle M^{\leq d-1} \cup\left(\mathbb{T}_{\leq d-1}^{n} \backslash U\right)\right\rangle_{K} \cap I} .
$$

Therefore for a fixed $U \subseteq \mathbb{T}_{\leq d-1}^{n}$, the inequality (3.6c) is equivalent to (3.1c) with $U$ replaced by its complement $\mathbb{T}_{\leq d-1}^{n} \backslash U$. The equivalence of (3.6c) and (3.1c) stated for all subsets $U$ follows, noting that $\left|M^{\leq d-1}\right|=\left|\mathbb{T}_{\leq d-1}^{n}\right|-d$, as shown at the beginning of the proof.

\section{COMPUTING BORDER BASES USING THE ORDER IDEAL POLYTOPE}

In the following we explain how Theorem 3.2 can be used to actually compute border bases for general order ideals. We cannot expect to be able to compute a border basis for any order ideal, simply as such a basis does not necessarily exist. As a priori it is unclear which are the admissible order ideals $\mathcal{O}$ for an ideal $I$ given by generators, we use an indirect way to specify $\mathcal{O}$ : we use a weight vector $w \in \mathbb{R}^{\mathbb{T}^{n}}$ and want to find $\mathcal{O} \in \Lambda(I)$ maximizing the total weight $\sum_{m \in \mathcal{O}} w_{m}$ of $\mathcal{O}$. Note that any admissible order ideal $\mathcal{O}$ can be specified via an appropriate weight vector $w$ so our approach, while indirect, is without loss of generality. As $w$ is an infinite vector, in practice it should be probably given explicitly for a finite number of coordinates, and the remaining coordinates are declared to be 0 or some other fixed value; this is not a restriction as all admissible order ideals are finite and the occuring maximum degree is bounded. Recall that $\Lambda(I)$ denotes the set of all admissible order ideals of $I$. We will show how to compute such a weight-maximal $\mathcal{O}$ and its border basis for a zero-dimensional ideal $I \subseteq K[\mathbb{X}]$.

We adapt the border basis algorithm in [35].

Algorithm 4.1 (Generalized border basis algorithm-BBasis).

Input: $F$ a finite generating set of a zero-dimensional ideal, and a weight vector $w$ on $\mathbb{T}^{n}$. Output: $\mathcal{G}$ a border basis of the ideal.

(1) Let $d:=\max _{f \in F} \operatorname{deg}(f)$.

(2) $M:=\operatorname{LStabSpan}\left(F, \mathbb{T}_{\leq d}^{n}\right)$ using a degree-compatible ordering on $\mathbb{T}_{\leq d}^{n}$ (i.e., $m_{1}<m_{2}$ whenever $\operatorname{deg} m_{1}<\operatorname{deg} m_{2}$ ).

(3) If $\mathbb{T}_{=d}^{n} \nsubseteq \mathrm{LT}(M)$ then set $d:=d+1$ and go to step (2).

(4) Set $d_{\text {old }}:=d, d:=\left|\mathbb{T}_{\leq d}^{n}\right|-|M|$. If $d \leq d_{\text {old }}$ then let $M:=M \leq d$. Otherwise let $M:=$ LStabSpan $\left(F, \mathbb{T}_{\leq d}^{n}\right)$.

(5) Write up the system (3.6) of inequalities for $M$ and $d$. Choose an integral solution $z$ maximizing wz. Set $\mathcal{O}:=\left\{m \in \mathbb{T}_{\leq d-1}^{n}: z_{m}=1\right\}$.

(6) Let $\mathcal{G}:=$ Basistransformation $(M, \mathcal{O})$.

Our generalized border basis algorithm 4.1 first determines the right computational universe $\mathbb{T}_{\leq d}^{n}$ until step (4), i.e., a large enough $d \in \mathbb{N}$ such that the associated $\mathbb{T}_{<d}^{n}$-stabilized span $M$ contains all border bases. Here step (3) is a convenient way to quickly check whether the universe is already large enough. Step (4)] adjusts $d$ to the actual dimension of $K[\mathbb{X}] / I$ and adjusts $M$.

In the second phase, step (5)] optimizes over the order ideal polytope $P\left(\langle M\rangle_{K[\mathbb{X}]}\right)$ to find an optimal admissible order ideal using a mixed integer programming solver, and then step (6) computes the corresponding border basis. The main idea of this last step is to apply Gaussian elimination to $M$ to bring it into a form where with the exception of the leading terms, all monomials are from $\mathcal{O}$.

Lemma 4.2. Let $L=\mathbb{T}_{\leq \ell}^{n}$ with $\ell \in \mathbb{N}$, let $M$ be a non-empty finite set of polynomials satisfying $\langle M\rangle_{K}=$ $\langle M\rangle_{K[\mathbb{X}]} \cap\langle L\rangle_{K}$ and let $\mathcal{O}$ be an order ideal with $\partial \mathcal{O} \subseteq L$ and $\mathcal{O} \in \Lambda\left(\langle M\rangle_{K[\mathbb{X}]}\right)$. Then Algorithm 4.3 returns an $\mathcal{O}$-border basis $\mathcal{G}$ of $\langle M\rangle_{K[\mathbb{X}]}$. 
Proof. First, because $M \subseteq L$ and $M$ is non-empty, clearly the largest degree is $\ell$ among the polynomials in $\langle M\rangle_{K}=\langle M\rangle_{K[\mathbb{X}]} \cap\langle L\rangle_{K}$ and hence in $M$. Thus step (1) computes the correct value of $\ell$.

As $\mathcal{O} \in \Lambda\left(\langle M\rangle_{K[\mathbb{X}]}\right)$ we have $K[\mathbb{X}]=\langle M\rangle_{K[\mathbb{X}]} \oplus\langle\mathcal{O}\rangle_{K}$ and hence

$$
\langle L\rangle_{K}=\langle L\rangle_{K} \cap\left(\langle M\rangle_{K[\mathbb{X}]} \oplus\langle\mathcal{O}\rangle_{K}\right)=\left(\langle L\rangle_{K} \cap\langle M\rangle_{K[\mathbb{X}]}\right) \oplus\langle\mathcal{O}\rangle_{K}=\langle M\rangle_{K} \oplus\langle\mathcal{O}\rangle_{K}
$$

by the modular law, in particular, $\left|\mathcal{G}^{\prime}\right|=|M|=|L|-|\mathcal{O}|$. Now, none of the polynomials in $\mathcal{G}^{\prime}$ are supported on $\mathcal{O}$, and as $\mathcal{O}$ is an initial segment of the ordering used for Gaussian elimination, it follows that all the leading terms in $\mathcal{G}^{\prime}$ lie in $L \backslash \mathcal{O}$. Since $\left|\mathcal{G}^{\prime}\right|=|M|=|L|-|\mathcal{O}|$, it follows that all $m \in L \backslash \mathcal{O}$ appear as leading term exactly once in $\mathcal{G}^{\prime}$, and hence not as other term, i.e., all polynomials $g \in \mathcal{G}^{\prime}$ have the form

$$
g=m_{0}-\sum_{m \in \mathcal{O}} a_{m} m, \quad m_{0} \in L \backslash \mathcal{O} .
$$

Obviously, restricting to the polynomials where the leading term is a border element of $\mathcal{O}$ in step (3) provides a border basis of $\mathcal{O}$.

Algorithm 4.3 (Basis transformation algorithm-Basistransformation).

Input: $M, \mathcal{O}$ as in Lemma 4.2

Output: $\mathcal{G}$ as in Lemma4.2

(1) Set $\ell:=\max _{m \in M} \operatorname{deg}(m)$.

(2) Reduce $M$ using Gaussian elimination (Algorithm 2.11) using an ordering where $\mathcal{O}$ is an initial segment (i.e., consists of the smallest elements): $\mathcal{G}^{\prime}:=\operatorname{GausSE} 1(M)$.

(3) Return $\mathcal{G}:=\left\{g \in \mathcal{G}^{\prime}: \operatorname{LT}(g) \in \partial \mathcal{O}\right\}$.

We will show now that Algorithm 4.1 computes an $\mathcal{O}$-border basis for $\mathcal{O} \in \Lambda(I)$.

Proposition 4.4. Let $F \subseteq K[\mathbb{X}]$ be a finite set of polynomials that generates a zero-dimensional ideal $I=$ $\langle F\rangle_{K[\mathbb{X}]}$. Then Algorithm 4.1] computes the $\mathcal{O}$-border basis $\mathcal{G}$ of I for any (chosen) $\mathcal{O} \in \Lambda(I)$.

Proof. Till step (3), the algorithm step by step enlarges the computational universe $\mathbb{T}_{\leq d}^{n}$ via increasing $d$. Since $I$ is zero-dimensional, the test $\mathbb{T}_{=d}^{n} \subseteq \operatorname{LT}(M)$ will be true for large enough $d$, hence the algorithm will eventually reach step (4). By Corollary 2.15, we have then $\langle M\rangle_{K}=I \leq d$, and that step (4) sets $d$ to the dimension of $K[\mathbb{X}] / I$. It also updates $M$ so that together with the new $d$ it satisfies $\langle M\rangle_{K}=I \leq d$. Obviously, $\mathbb{T}_{\leq d}^{n}$ contains all order ideals supporting a border basis, i.e., all $\mathcal{O} \in \Lambda(I)$ and even the boundary of these order ideals. Observe that $I=\langle F\rangle_{K[\mathbb{X}]}=\langle M\rangle_{K[\mathbb{X}]}$ and thus, by Lemma 4.2, it follows that $\mathcal{G}$ is indeed an $\mathcal{O}$-border basis of $\langle F\rangle_{K[\mathbb{X}]}$. Note that $\partial \mathcal{O} \subseteq \mathbb{T}_{\leq d}^{n}$ follows via $\langle M\rangle_{K}^{\leq d} /\langle M\rangle_{K}^{\leq d-1} \cong\left\langle\mathbb{T}_{=d}^{n}\right\rangle_{K}$ as $d$ is the dimension of $K[\mathbb{X}] / I$.

We conclude that $M$ satisfies the conditions of Lemma 3.9, e.g., $d=\left|\mathbb{T}_{<d}^{n}\right|-|M|$ is ensured by step (4), and therefore the integral solutions of the system used in step (5) are all the characteristic vectors of admissible order ideals. This step therefore really computes a maximal-weight admissible order ideal $\mathcal{O}$. Finally, step (6) returns a border basis of $\mathcal{O}$ by Lemma 4.2.

The border basis algorithm in [35] allows using computational universes $L$ smaller than $\mathbb{T}_{\leq d}^{n}$, improving performance of the algorithm. However, as we want to consider all order ideals and border bases, we deliberately chose the computational universe large enough to contain all possible order ideals. If a subset of all admissible order ideals is sufficient, then the same optimizations can be applied throughout.

For certain choices of the weight vector $w$ though it can be hard to compute a maximum weight order ideal as we will show now. In fact this also shows that there is no general, efficient way of specifying any admissible order ideal (unless coNP $=\mathrm{NP}$ ). 


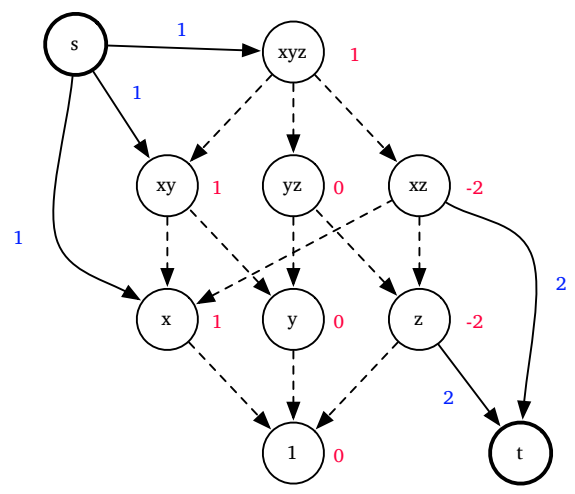

Figure 5.1. Order ideal computation as minimum cut problem. In this example, the order ideal consists of all monomials dividing $x y z$, i.e., $\{1, x, y, z, x y, y z, x z, x y z\}$, with (arbitrarily) chosen weights $\{0,1,0,-2,1,0,-2,1\}$. The weight $w_{u}$ of a monomial $u$ is shown in red next to the node of the monomial. Arcs are labelled in blue with their capacity resulting from the weights of monomials. Arcs with capacity $\infty$ are dashed. Arcs with capacity 0 are omitted.

\section{COMPLEXITY OF FINDING MAXIMUM WEIGHT ORDER IDEALS}

In this section, we show that finding a maximum weight, admissible order ideal of a zerodimensional ideal given by generators is NP-hard (Theorem 5.1). The hardness result is unexpected in the sense that we merely ask for a nice basis transformation. On the other hand it highlights the crucial role of order ideals in describing the combinatorial structure of the ideal. As an immediate consequence it follows that it is rather unlikely that we can obtain a good characterization of the integral hull of the order ideal polytope $P\left(\langle M\rangle_{K[\mathbb{X}]}\right)$ (unless NP $=$ coNP) and we will not be able to compute order ideals that support a border basis and have maximum weight efficiently in the worst case (unless NP $=\mathrm{P}$ ). This shows that it is hard not only to compute the necessary liftings of the initial set of polynomials via the LStabSpan procedure but also to actually determine an optimal choice of an order ideal once an L-stable span has been computed.

From a practical point of view this is not too problematic as, although NP-hard, computing a maximum weight order ideal is no harder than actually computing the LStabSpan in general. For bounds on the degree $d \in \mathbb{N}$ needed to compute border bases, see e.g., [22, Lemma 2.4]; the border basis algorithm generates the Nullstellensatz certificates and is therefore subject to the same bounds. Further, state-of-the-art mixed integer programming solvers such as scip [2], cplex [19], or gurobi [28] can handle instance sizes far beyond the point for which the actual border bases can be computed. Very good solutions can also be generated using simple local search schemes starting from a feasible order ideal derived from a degree-compatible term ordering.

5.1. Fast without constraint. Determining an order ideal of maximum weight (not necessarily supporting a border basis!) in a computational universe $L$ without having any constraints on the dimension of the respective spaces can be done in time polynomial in $|L|$ as we will show now. This follows with [47] and we simply transform the maximum weight order ideal problem into a minimum cut problem. For this let $w \in \mathbb{Z}^{L}$ be a weight vector. We define a directed graph $\Gamma:=(V, A)$ with $V:=L \cup\{s, t\}$ and $\tilde{A}:=\{(u, v) \mid u, v \in L$ and $v \mid u\}$, i.e., whenever $v \mid u$ we add an arc from $u$ to $v$. In fact, it is enough to have an arc when $u=v x$ for some variable $x$, i.e., to consider the transitive reduction of $\tilde{A}$. Define

$$
A:=\tilde{A} \cup\left\{(s, u) \mid u \in L, w_{u}>0\right\} \cup\left\{(u, t) \mid u \in L, w_{u}<0\right\} .
$$

Now we turn to the arc capacities. Let $\kappa(u, v)$ denote the capacity or arc $(u, v)$ defined as follows. For $u$ and $v$ both in $L$, we set $\kappa(u, v):=\infty$. We set $\kappa(s, v):=w_{v}$ and $\kappa(u, t)=-w_{u}$ for $u, v$ in $L$. An example is depicted in Figure 5.1. 
For $U, W \subseteq V$, we define $C(U, W):=\sum_{(u, w) \in U \times W} \kappa(u, w)$ as the directed cut value. An $(s, t)$-cut $(S, \bar{S})$ is a partition $S \dot{\cup}=V$ of the vertices of $V$ with $s \in S$ and $t \in \bar{S}$ and the weight of the cut is $C(S, \bar{S})$; note that the direction of the arcs matters. We would like to compute an order ideal contained in $L$ with maximum weight:

$$
\max \left\{\sum_{u \in \mathcal{O}} w_{u} \mid \mathcal{O} \subseteq L \text { order ideal }\right\} .
$$

Observe that $(S, \bar{S})$ is a directed cut in $\Gamma$ of finite weight, if and only if there exists no $\operatorname{arc}(u, v) \in \tilde{A}$ with $u \in S$ and $v \in \bar{S}$, i.e., for all monomials $u, v \in \mathcal{O}$ with $v \mid u$, if $u \in S$ then $v \in S$. In other words, $(S, \bar{S})$ is a cut in $\Gamma$ of finite weight if and only if $S \backslash\{s\}$ is an order ideal. We can therefore rewrite the optimization problem as follows:

$$
\begin{aligned}
\max & \left\{\sum_{u \in \mathcal{O}} w_{u} \mid \mathcal{O} \subseteq L \text { order ideal }\right\} \\
& =\max \{C(\{s\}, \mathcal{O})-C(\mathcal{O},\{t\}) \mid \mathcal{O} \subseteq L \text { order ideal }\} \\
& =\max \{C(\{s\}, L)-C(\{s\}, L \backslash \mathcal{O})-C(\mathcal{O},\{t\}) \mid \mathcal{O} \subseteq L \text { order ideal }\} \\
& =C(\{s\}, L)-\min \{C(\{s\}, L \backslash \mathcal{O})+C(\mathcal{O},\{t\}) \mid \mathcal{O} \subseteq L \text { order ideal }\} \\
& =C(\{s\}, L)-\min \{C(\{s\} \cup \mathcal{O},(L \backslash \mathcal{O}) \cup\{t\}) \mid \mathcal{O} \subseteq L\} .
\end{aligned}
$$

The last line asks for a minimum weight cut in the graph $\Gamma$. Note that we can indeed drop the condition that $\mathcal{O}$ has to be an order ideal as it is guaranteed implicitly by all finite weight cuts as explained above. The minimum cut can now be computed in polynomial time in the number of vertices and arcs (see e.g., [52]) and so can an order ideal $\mathcal{O}$ of maximum weight efficiently.

5.2. NP-hard with constraints. So far we did not include the additional requirements as specified by the order ideal polytope (see (3.6)), in order to obtain order ideals that do actually support a border basis of the ideal I under consideration. We will now show that when including these additional requirements, the problem of computing an order ideal of maximum weight becomes NP-hard. In [30, Discussion after Definition 3.2] it was indicated that determining a maximum weight order ideal of a pre-defined size is NP-hard by a reduction from MaxClique, however this is different from our problem, as we have additional constraints coming from the dimension of the factor spaces of the ideal (see constraints $(3.6 \mathrm{C})$ ).

We will show NP-hardness by a reduction from the $k$-CLIQUE problem, which is well known to be NP-complete (see, e.g., [27] or [20, GT22]). Given an undirected simple graph $\Gamma=(V, E)$, recall that a clique $C$ is a subset of $V$ such that for all distinct $u, v \in C$ we have $(u, v) \in E$. We consider the decision problem:

k-Clique: Let $\Gamma=(V, E)$ be an undirected simple graph. Decide whether $\Gamma$ contains a clique $C$ of size $k$.

Our optimization problem of interest is:

MAXIMUM WEIGHT ADMISSIBLE ORDER IDEAL: Let $M \subseteq K[\mathbb{X}]$ be a system of polynomials generating a zerodimensional ideal and let $w \in \mathbb{Z}^{\mathbb{T}^{n}}$ be a weight on the monomials. Compute an admissible order ideal $\mathcal{O} \subseteq \mathbb{T}^{n}$ for $\langle M\rangle_{K[\mathbb{X}]}$ with maximum weight $\sum_{m \in \mathcal{O}} w_{m}$ with respect to w, i.e., compute

$$
\underset{\mathcal{O} \in \Lambda\left(\langle M\rangle_{K[\mathbb{X}]}\right)}{\operatorname{argmax}} \sum_{m \in \mathcal{O}} w_{m}
$$

By a reduction from $k$-CLIQUE we obtain:

Theorem 5.1. MAXIMUM WEIGHT ADMISSIBLE ORDER IDEAL is NP-hard over ground fields K of characteristic 0 . 
As a preparation for the proof, we show that for every graph $\Gamma=(V, E)$ and $k \in[|V|]$ there exists a system of polynomials $F_{|V|, k} \subseteq K\left[x_{v} \mid v \in V\right]$ spanning a zero-dimensional ideal such that solving the MAXIMUM WEIGHT ADMISSIBLE ORDER IDEAL problem for $F_{|V|, k}$ solves the $k$-CLIQUE problem for $\Gamma$. For this, we construct an ideal encoding all $k$-cliques of the complete graph on $n$ vertices: Let $n \in \mathbb{N}$ and $k \in[n]$ and define

$$
F_{n, k}:=\left\{v_{j} \mid j \in[n-k]\right\} \cup \mathbb{T}_{=3}^{n}
$$

with $v_{j}:=\sum_{i \in[n]} i^{j} x_{i}$. We consider the ideal generated by $F_{n, k}$. We show that its order ideals are in one-to-one correspondence with the $k$-element subsets of the set of $n$ variables $x_{1}, \ldots, x_{n}$ as stated in the following lemma.

Lemma 5.2. Let $K$ be a field of characteristic 0 together with $n \in \mathbb{N}$ and $k \in[n]$. Then $F_{n, k}$ generates a zero-dimensional ideal such that $\mathcal{O} \in \Lambda\left(\left\langle F_{n, k}\right\rangle_{K[\mathbb{X}]}\right)$ if and only if $\mathcal{O}^{=1} \subseteq \mathbb{T}_{=1}^{n}$ with $\left|\mathcal{O}^{=1}\right|=k$, $\mathcal{O}^{=2}=\left\{x y \mid x, y \in \mathcal{O}^{=1}\right\}$, and $\mathcal{O}^{=\ell}=\varnothing$ for all $\ell \geq 3$.

Proof. We start by providing an explicit representation of the factor ring $K\left[x_{1}, \ldots, x_{n}\right] /\left\langle F_{n, k}\right\rangle_{K[\mathbb{X}]}$. As $F_{n, k}$ consists of homogeneous polynomials, it generates a homogeneous ideal $I$, and induces a degree decomposition of the factor ring:

$$
\frac{K\left[x_{1}, \ldots, x_{n}\right]}{\left\langle F_{n, k}\right\rangle_{K[\mathbb{X}]}}=\bigoplus_{i=0}^{\infty} \frac{\left\langle\mathbb{T}_{=i}^{n}\right\rangle_{K}}{I^{=i}} .
$$

To actually determine the factors, let $x_{i_{1}}, \ldots, x_{i_{k}}$ be $k$ many distinct variables from $x_{1}, \ldots, x_{n}$. We prove that $x_{i_{1}}, \ldots, x_{i_{k}}, v_{1}, \ldots, v_{n-k}$ is a vector space basis of $\left\langle\mathbb{T}_{=1}^{n}\right\rangle_{K}$ by showing that its coefficient matrix in the standard basis $x_{1} . \ldots, x_{n}$ has non-zero determinant. Expanding the determinant by the $k$ rows of $x_{i_{1}}, \ldots, x_{i_{k}}$, each of which contains only one non-zero element, the determinant becomes equal to up to a sign to the Vandermonde matrix of the set of numbers $[n] \backslash\left\{i_{1}, \ldots, i_{k}\right\}$, and hence it is indeed non-zero.

The ring $K[\mathbb{X}]$ is also a polynomial ring in any basis of $\left\langle\mathbb{T}_{=1}^{n}\right\rangle_{K^{\prime}}$, and the basis $x_{i_{1}}, \ldots, x_{i_{k}}, v_{1}, \ldots$, $v_{n-k}$ is particularly suitable to determine the factor $K[\mathbb{X}] / I$ together with the degree decomposition:

$$
\begin{aligned}
\frac{K\left[x_{1}, \ldots, x_{n}\right]}{\left\langle F_{n, k}\right\rangle_{K[\mathbb{X}]}} & =\frac{K\left[x_{i_{1}}, \ldots, x_{i_{k}}, v_{1}, \ldots, v_{n-k}\right]}{\left\langle v_{1}, \ldots, v_{n-k}, \mathbb{T}_{=3}^{n}\right\rangle_{K[\mathbb{X}]}} \\
& =\frac{K\left[x_{i_{1}}, \ldots, x_{i_{k}}\right]}{\left\langle\mathbb{T}_{=3}^{n}\right\rangle_{K[\mathbb{X}]}}=\langle 1\rangle_{K} \oplus\left\langle x_{i_{1}}, \ldots, x_{i_{k}}\right\rangle_{K} \oplus\left\langle x_{i} x_{j}: i, j \in\left\{i_{1}, \ldots, i_{k}\right\}\right\rangle_{K}
\end{aligned}
$$

where the generating sets are actually bases of the respective degree components.

Given an order ideal $\mathcal{O}$ of $I$, the isomorphism $\langle\mathcal{O}\rangle_{K} \cong K[\mathbb{X}] / I$ clearly preserves the degree decomposition, i.e., $\left\langle\mathcal{O}^{=\ell}\right\rangle_{K} \cong(K[\mathbb{X}] / I)^{=\ell}$ for all $\ell$. Hence $\left|\mathcal{O}^{=0}\right|=1,\left|\mathcal{O}^{=1}\right|=k,\left|\mathcal{O}^{=2}\right|=\left(\begin{array}{c}k+1 \\ 2\end{array}\right)$, and $\left|\mathcal{O}^{=\ell}\right|=0$ for $\ell \geq 3$. It follows that $\mathcal{O}$ has the claimed form, in particular, $\mathcal{O}^{=2}=\{x y \mid x, y \in$ $\mathcal{O}^{=1}$ \} as the left-hand side is clearly a subset of the right-hand side, and they have the same finite size.

For the other direction, let $\mathcal{O}^{=1}=\left\{x_{i_{1}}, \ldots, x_{i_{k}}\right\}$ with $\mathcal{O}^{=2}=\left\{x y \mid x, y \in \mathcal{O}^{=1}\right\}, \mathcal{O}^{=0}=\{1\}$ and $\mathcal{O}^{=\ell}=\varnothing$ for $\ell \geq 3$. Then $\mathcal{O}$ is an order ideal, and (5.1) shows that the image of $\mathcal{O}$ in $K[\mathbb{X}] / I$ is a basis. Thus $\mathcal{O}$ is an admissible order ideal for $I$, as claimed.

Note that the order ideals of $F_{n, k}$ indeed correspond to the $k$-cliques of the complete graph on $n$ vertices: If $\mathcal{O} \in \Lambda\left(F_{n, k}\right)$, then $\mathcal{O}^{=1}=\left\{x_{i_{1}}, \ldots, x_{i_{k}}\right\}$ and $x_{i_{j}} x_{i_{l}} \in \mathcal{O}^{=2}$ if and only if $x_{i_{j}}, x_{i_{l}} \in \mathcal{O}^{=1}$. If 
we now remove all elements of the form $x_{i_{j}}^{2}$ with $x_{i_{j}} \in \mathcal{O}^{=1}$, and there are $k$ of those, then

$$
\left|\mathcal{O}^{=2} \backslash\left\{x_{i_{j}}^{2} \mid x_{i_{j}} \in \mathcal{O}^{=1}\right\}\right|=\frac{k(k-1)}{2},
$$

the size of a $k$-clique. We are ready to prove the main result of this section.

Proof of Theorem 5.1. The proof is by a reduction from the NP-hard $k$-CLIQUE problem. Let us start with an instance of $k$-CLIQUE, i.e., an undirected graph $\Gamma=(V, E)$ with $n:=|V|$ and $k \in[n]$. We consider $M:=F_{n, k}$ and define $w \in \mathbb{Z}^{\mathbb{T}^{n}}$ via

$$
w_{m}= \begin{cases}1, & \text { if } m=x_{u} x_{v} \text { and either }(u, v) \in E \text { or } u=v ; \\ 0, & \text { otherwise, }\end{cases}
$$

for all $m \in \mathbb{T}^{n}$. By Lemma[5.2, there is a bijection of the admissible order ideals $\mathcal{O}$ of $\langle M\rangle_{K[\mathbb{X}]}$ and the $k$-cliques of the complete graph on $n$ vertices given by

$$
C_{\mathcal{O}}:=\left\{v \in V \mid x_{v} \in \mathcal{O}\right\}
$$

The weight of $\mathcal{O}$ is the sum of the weights of the monomials $x_{u} x_{v}$ in $\mathcal{O}$. To the weight of $\mathcal{O}$, the contribution of the monomials with $u=v$, i.e., of the form $x_{v}^{2}$ is the number of vertices of $C_{\mathcal{O}}$, i.e., $k$. The monomials $x_{u} x_{v}$ with $u \neq v$ contribute the number of edges in $C_{\mathcal{O}} \cap \Gamma$ to the weight of $\mathcal{O}$. Hence the weight of $\mathcal{O}$ is the sum of $k$ and the number of edges in $C_{\mathcal{O}} \cap \Gamma$.

The largest possible value of this weight is $k(k+1) / 2$, and this is realized exactly by cliques $C_{\mathcal{O}}$ of $\Gamma$ of size $k$. (If such cliques do not exist, then the maximal weight is less than $k(k+1) / 2$.) All in all, the maximum weight is $k(k+1) / 2$ if and only if $\Gamma$ contains a clique $C_{\mathcal{O}}$ of size $k$. We obtain that MAXIMUM WEIGHT ADMISSIBLE ORDER IDEAL solves $k$-CLIQUE and so the former has to be NP-hard.

5.3. Extension complexity of admissible order ideals. The order ideal polytope $P(I)$ was introduced as a relaxation of the convex hull OIP $(I)$ of (the characteristic vectors of) all admissible order ideals of the ideal $I$. Therefore one might wonder whether there exists a description with a polynomial number of linear inequalities of the convex hull $\operatorname{OIP}(I)$. This question is the natural counterpart of algorithmic complexity in the context of linear programming. Here we show that in general OIP $(I)$ requires a subexponential number of inequalities in the size of the computational universe, even if one allows additional extra variables, i.e., the extension complexity (see below) of $\mathrm{OIP}(I)$ is subexponential. As customary in extended formulations this result does not depend on any complexity theoretic assumptions, see [13, 16, 26, 32] for details. The result could be also formulated independent of the order ideal polytope, namely, that the linear programming formulation complexity (complexity measured in the size of a linear program) of the combinatorial problem to find a maximum-weight admissible order ideal is subexponential. However, for simplicity, we stick to the polyhedral formulation, and refer the interested reader to [13] for the general model.

Recall that the extension complexity $\mathrm{xc}(P)$ of a polyhedron $P$ is the minimum number of facets of a polyhedron $Q$, such that $P$ is an affine image of $Q$. The extension complexity captures the inherent complexity of a polytope being expressed by means of linear inequalities.

Theorem 5.3. For any ground field $K$ of characteristic 0 , there is an ideal I of $K\left[x_{1}, \ldots, x_{2 n}\right]$ such that all admissible order ideals of I contain monomials only up to degree 2 , and

$$
\mathrm{xc}(\mathrm{OIP}(I))=2^{\Omega(n)} .
$$

Proof. We shall use the ideal $I$ generated by $F_{2 n, n}$ from Lemma 5.2, whose admissible order ideals have a nice description, and all of which consist of monomials only up to degree 2 . Therefore we 
obtain the following description of $\operatorname{OIP}(I)$ :

$$
\begin{aligned}
\mathrm{OIP}(I) & =\operatorname{conv}\left\{y^{S}: S \subseteq[2 n],|S|=n\right\} \subseteq[0,1]^{\mathbb{T}^{n}} \\
y_{x_{i} x_{j}}^{S} & = \begin{cases}1, & \text { if } i, j \in S, \\
0, & \text { otherwise. }\end{cases}
\end{aligned}
$$

Here for simplicity we restrict to the relevant coordinates only, the other coordinates are affine combinations of these (e.g., $\left.y_{x_{i}}=y_{x_{i}^{2}}, y_{1}=0\right)$ ). We will show that the correlation polytope is an affine projection of $\operatorname{OIP}(I)$, and therefore $\mathrm{xc}(\operatorname{OIP}(I)) \geq \mathrm{xc}(\operatorname{COR}(n))=2^{\Omega(n)}$ by [26, Lemma 9(i)] and [10, Theorem 4(i)].

Recall that the correlation polytope is the convex hull of all 0/1-matrices of rank 1 :

$$
\begin{aligned}
\operatorname{COR}(n) & :=\operatorname{conv}\left\{\mathbb{1}_{S} \mathbb{1}_{S}^{\top} \mid S \subseteq[n]\right\} \subseteq[0,1]^{n \times n}, \\
\mathbb{1}_{S}(i) & := \begin{cases}1, & \text { if } i \in S, \\
0, & \text { otherwise. }\end{cases}
\end{aligned}
$$

An affine projection $f: \operatorname{OIP}(I) \rightarrow \operatorname{COR}(n)$ is clearly provided by

$$
f(y)_{i, j}:=y_{x_{i} x_{j}} \quad i, j \in[n],
$$

where the vertices of $\operatorname{OIP}(I)$ are mapped to vertices of $\operatorname{COR}(n)$

$$
f\left(y^{S}\right)=\mathbb{1}_{S \cap[n]} \mathbb{1}_{S \cap[n]}^{\top} \quad S \subseteq[2 n],|S|=n .
$$

Note that $2 n$ variables were chosen for $\operatorname{OIP}(I)$ so that every subset $T \subseteq[n]$ arises as an intersection $T=S \cap[n]$ for some $S \subseteq[2 n]$ of size $[n]$.

5.4. Discussion of the complexity of finding maximal weight admissible order ideals. We now briefly summarize the implications of these complexity results. Note that the hardness proof in Section 5.2 is independent of the order ideal polytope, and shows worst-case hardness for any algorithm.

(1) No general characterization of all admissible order ideals. The hardness in Section 5.2 is established for the MAXIMUM WEIGHT ADMISSIBLE ORDER IDEAL problem. As such, unless NP = coNP, which is generally believed to be not the case, in general there will be no good characterization of order ideals that will be admissible for a given ideal. Complementing this, the result in Section 5.3 rules out any small linear programming formulation for the convex hull of admissible order ideals irrespective of NP vs. coNP.

(2) No theoretically efficient algorithm for computing maximal weight order ideals. In particular, unless $\mathrm{NP}=\mathrm{P}$, there will be no polynomial time algorithm computing a maximal weight order ideal. However, this is worst-case complexity, and does not necessarily capture well real-world performance, as e.g., the Traveling Salesman Problem is also NP-hard, however solvable for real-world instances with millions of cities in reasonable computational time (see e.g., [7]).

(3) Real-world computational complexity. While the determinination of a maximal weight admissible order ideal is theoretically NP-hard as discussed above, in practice this problem can be solved very easily with state-of-the-art solvers such as e.g., SCip, CPLEX, or Gurobi, typically in the order of seconds. Comparing the generalized border basis algorithm (Algorithm 4.1) to the border basis algorithm in [35], the major difference is the additional computational steps (5) and (6), The basis transformation in (6) is very cheap, and so is step (5) for all practical purposes as indicated. 
The real bottleneck in our border basis algorithm (and also the one in [35], upon which ours is based) is computing the $L$-stable span, which can be several orders of magnitude slower than determining the order ideal. In summary, we believe that our method has little additional costs compared to the border basis algorithm in [35], when incorporated correctly into a state-of-the-art implementation.

(4) Size of the order ideal polytope. The description of the order ideal polytope in Definition 3.1 has a number of inequalities of roughly $O\left(n^{d^{2}}\right)$ due to (3.1c). This is roughly the largest possible number of facets of the order ideal polytope, i.e., the number of all order ideals. Recall that the order ideal polytope is not necessarily the convex hull of all admissible order ideals, but only a relaxation of it, and as Theorem 5.3 shows (where $d=2$ ), the convex hull itself requires much more inequalities in the worst case. However, it is conceivable that the convex hull admits an even smaller relaxation than the order ideal polytope via uncapacitated network flows or separating the inequalities (see e.g., [50]) as e.g., done for the spanning tree polytope.

\section{Computational Results}

We performed computational tests to verify the practical feasibility of our method, with an emphasis of the optimization step over the order ideal polytope, once it is written down, as this is the new aspect in our algorithm. Because this is not expected to be the bottleneck, we refrained from a comprehensive performance test, and used small-sized problems.

For simplicity, we computed only degree-compatible order ideals. All computations were performed with COCOA 4.7.5 [15] and scip 1.1.0 [2] on a $2 \mathrm{Ghz}$ Dual Core Intel machine with 2 GB of main memoryt.

Test setup. The employed methodology was as follows. We first computed a border basis using the border basis algorithm in [35]. From the last run of the algorithm we extracted the $L$-stabilized span and brought it into canonical form as the actual $L$-stable span computation is not the focus here but the computation of admissible order ideals. We then generated the constraint (3.6b) from the order ideal that we obtained; from the L-stabilized span in matrix from, we generated the constraints (3.6C) adapted to degree-compatible order ideals. We performed computations on various sets of systems of polynomial equations. We then transcribed these constraints into the CPLEX LP format which served as input for scip. For the optimization we chose various weight vectors. We tested random weight vectors and we constructed a weight vector with the intent to make the optimization particularly hard by giving monomials deep in the order ideal negative weights and assigning positive weights for the outer elements.

Results. We report the results of our tests in Table 3, In all cases, the optimization (i.e., the computation of the maximum weight order ideal) was performed in less than a second (see column optimization), whereas the actual calculation of the initial border bases was significantly more time consuming. This is not unexpected as the computation of the $L$-stable span is significantly more involved than computing a maximum weight order ideal: the former can be double exponential whereas the latter is at most single exponential via complete enumeration.

An example application: counting order ideals. When computationally feasible, we also counted all feasible order ideals with scip, which basically means enumerating all feasible solutions, to demonstrate feasibility for reasonably sized instances. This is reported in column counting.

\footnotetext{
${ }^{1}$ Source code is available at: https://app.box.com/s/fcxtocvpqqj0b2dezj40v4tn24sfkk1m
} 


\begin{tabular}{|c|c|c|c|c|}
\hline polynomial system & $\begin{array}{l}\text { degree vector of } \\
\text { order ideal }\end{array}$ & optimization [s] & counting $[\mathrm{s}]$ & \# order ideals \\
\hline$x^{3}, x y^{2}+y^{3}$ & $(1,2,3,2,1)$ & $\overline{<<0.01}$ & $\overline{0.02}$ & $\overline{\overline{3}}$ \\
\hline $\begin{array}{c}\text { vanishing ideal of the } \\
\text { points }(0,0,0,1), \\
(1,0,0,2),(3,0,0,2), \\
(5,0,0,3),(-1,0,0,4), \\
(4,4,4,5),(0,0,7,6)) .\end{array}$ & $(1,4,2)$ & $<0.01$ & 0.02 & 45 \\
\hline $\begin{array}{c}x+y+z-u-v \\
x^{2}-x, y^{2}-y, z^{2}-z \\
u^{2}-u, v^{2}-v\end{array}$ & $(1,4,5)$ & $<0.01$ & 0.35 & 1,260 \\
\hline $\begin{array}{c}x+y+z-u-v \\
x^{3}-x, y^{3}-y, z^{2}-z \\
u^{2}-u, v^{2}-v\end{array}$ & $(1,4,7,6)$ & 0.02 & 51.50 & 106,820 \\
\hline $\begin{array}{c}x+y+z-u-v \\
x^{3}-x, y^{3}-y, z^{3}-z \\
u^{2}-u, v^{2}-v\end{array}$ & $(1,4,8,9)$ & 0.02 & 53.00 & 108,900 \\
\hline $\begin{array}{c}x+y+z-u-v \\
x^{3}-x, y^{3}-y, z^{3}-z \\
u^{3}-u, v^{2}-v\end{array}$ & $(1,4,9,12,9)$ & 0.08 & $300.00^{*}$ & $>1,349,154$ \\
\hline $\begin{array}{c}x+y+z-u-v+a \\
x^{2}-x, y^{2}-y, z^{2}-z \\
u^{2}-u, v^{2}-v, a^{2}-a\end{array}$ & $(1,5,9)$ & $<0.01$ & 8.68 & 30,030 \\
\hline
\end{tabular}

TABLE 3. Computational results. The first column contains the considered polynomial system. The second column contains the degree vector of the order ideal, i.e., (dim $\left.I^{\leq i} / I^{\leq i-1}\right)_{i}$ starting with $i=0$ and $I^{\leq-1}:=0$. The third column contains the average time (in seconds) needed to optimize a random weight over the order ideal polytope (we performed 20 runs for each system). The fourth column contains the time (in seconds) needed to count all admissible degree-compatible order ideals and the last column contains the actual number of admissible degree-compatible order ideals. The ${ }^{\prime * \prime}$ indicates that the counting had been stopped after 300 seconds. The number of order ideals reported in this case is the number that have been counted up to that point in time.

\section{CONCLUding REMARKS}

We gave a polyhedral characterization of all order ideals that support a border basis of a given zero-dimensional ideal. While it is impossible to provide a full linear description of polynomial size of the integral hull contained in the order ideal polytope due to Theorem [5.3 it might be possible to obtain a compact extended formulation of the order ideal polytope itself (not its integral hull). We leave this as an open question.

\section{REFERENCES}

[1] J. Abbott, C. Fassino, and M.-L. Torrente. Stable border bases for ideals of points. Journal of Symbolic Computation, 43:883-894, 2008.

[2] T. Achterberg. SCIP: solving constraint integer programs. Mathematical Programming Computation, 1(1):1-41, 2009.

[3] N. Alon. Combinatorial Nullstellensatz. Combinatorics, Probability and Computing, 8:7-29, 1999.

[4] N. Alon, M.B. Nathanson, and I.Z. Ruzsa. The polynomial method and restricted sums of congruence classes. Journal of Number Theory, 56:404-417, 1996.

[5] Prabhanjan V Ananth and Ambedkar Dukkipati. Complexity of Gröbner basis detection and border basis detection. Theoretical Computer Science, 459:1-15, 2012. 
[6] Prabhanjan Vijendra Ananth and Ambedkar Dukkipati. Border basis detection is NP-complete. In Proceedings of the 36th international symposium on Symbolic and algebraic computation, pages 11-18. ACM New York, NY, USA, 2011.

[7] David L Applegate, Robert E Bixby, Vasek Chvatal, and William J Cook. The Traveling Salesman Problem: A Computational Study: A Computational Study. Princeton university press, 2011.

[8] W. Auzinger and H.J. Stetter. An elimination algorithm for the computation of all zeros of a system of multivariate polynomial equations. In Proceedings of the International Conference on Numerical Mathematics, pages 11-30. National University of Singapore, May 31-June 4, 1988, Birkhäuser, 1988.

[9] M. Borges-Quintana, M.A. Borges-Trenard, and E. Martínez-Moro. An application of Möller's algorithm to coding theory. In M. Sala, T. Mora, L. Perret, S. Sakata, and C. Traverso, editors, Gröbner Bases, Coding, and Cryptography, pages 379-384. Springer, 2009.

[10] G. Braun, S. Fiorini, S. Pokutta, and D. Steurer. Approximation limits of linear programs (beyond hierarchies). Mathematics of Operations Research, 2014. DOI: 10.1287/moor.2014.0694. arXiv:1204.0957, doi:10.1287/moor.2014.0694.

[11] G. Braun and S. Pokutta. Border bases and order ideals: a polyhedral characterization. arXiv:0912.1502, 2009.

[12] G. Braun and S. Pokutta. A polyhedral approach to border bases. arXiv:0911.0859, 2009.

[13] G. Braun, S. Pokutta, and D. Zink. Inapproximability of combinatorial problems via small LPs and SDPs. Proceeedings of STOC, 2015.

[14] Marta Abril Bucero and Bernard Mourrain. Border basis relaxation for polynomial optimization. arXiv preprint arXiv:1404.5489, 2014.

[15] CoCoA Team. CoCoA: a system for doing computations in commutative algebra, 2009. Available from: http://cocoa.dima.unige.it

[16] M. Conforti, G. Cornuéjols, and G. Zambelli. Extended formulations in combinatorial optimization. 4OR, 8:1-48, 2010. doi:10.1007/s10288-010-0122-z.

[17] N. Courtois, A. Klimov, J. Patarin, and A. Shamir. Efficient algorithms for solving overdefined systems of multivariate polynomial equations. Lecture Notes in Computer Science, 1807:392-407, 2000.

[18] D. Cox, J. Little, and D. O'Shea. Ideals, Varieties, and Algorithms: An Introduction to Computational Algebraic Geometry and Commutative Algebra. Springer, 2007.

[19] CPLEX. 11.0 User's Manual. ILOG SA, Gentilly, France, 2008.

[20] P. Crescenzi and V. Kann. A compendium of NP optimization problems. online manuscript, 1998.

[21] J.A. De Loera, J. Lee, P.N. Malkin, and S. Margulies. Hilbert's Nullstellensatz and an algorithm for proving combinatorial infeasibility. In Proceedings of the twenty-first international symposium on Symbolic and algebraic computation ISSAC'08, 2008.

[22] J.A. De Loera, J. Lee, S. Margulies, and S. Onn. Expressing Combinatorial Problems by Systems of Polynomial Equations and Hilbert's Nullstellensatz. Combinatorics, Probability and Computing, 18(4):551-582, 2009.

[23] J.A. De Loera, P.N. Malkin, and P.A. Parrilo. Mixed Integer Nonlinear Programming, volume 154 of The IMA Volumes in Mathematics and its Applications, chapter Computation with Polynomial Equations and Inequalities Arising in Combinatorial Optimization, pages 447-481. Springer New York, November 2012.

[24] Jesús A De Loera, Christopher J Hillar, Peter N Malkin, and Mohamed Omar. Recognizing graph theoretic properties with polynomial ideals. Elect. J. of Combinatorics, 17(1):R114, 2010.

[25] Jesús A De Loera, Jon Lee, Peter N Malkin, and Susan Margulies. Computing infeasibility certificates for combinatorial problems through Hilbert's Nullstellensatz. Journal of Symbolic Computation, 46(11):1260-1283, 2011.

[26] S. Fiorini, S. Massar, S. Pokutta, H. R. Tiwary, and R. de Wolf. Linear vs. semidefinite extended formulations: Exponential separation and strong lower bounds. Proceedings of STOC, 2012.

[27] M.R. Garey and D.S. Johnson. Computers and Intractability: A Guide to the Theory of NP-completeness. W.H. Freeman and Company, San Francisco, 1979.

[28] Gurobi. Gurobi 1.1.0 mixed integer linear programming solver. Gurobi Optmization Inc, 2009.

[29] D. Heldt, M. Kreuzer, S. Pokutta, and H. Poulisse. Approximate computation of zero-dimensional polynomial ideals. Journal of Symbolic Computation, 44(11):1566-1591, 2009. doi:10.1016/j • jsc. 2008.11 .010$.

[30] D.S. Hochbaum and A. Chen. Performance analysis and best implementations of old and new algorithms for the open-pit mining problem. Operations Research, pages 894-914, 2000.

[31] Gareth James, Daniela Witten, Trevor Hastie, and Robert Tibshirani. An introduction to statistical learning. Springer, 2013.

[32] V. Kaibel. Extended formulations in combinatorial optimization. Optima, 85:2-7, 2011.

[33] Stefan Kaspar. Computing border bases without using a term ordering. Beiträge zur Algebra und Geometrie/Contributions to Algebra and Geometry, 54(1):211-223, 2013.

[34] A. Kehrein and M. Kreuzer. Characterizations of border bases. Journal of Pure and Applied Algebra, 196:251-270, 2005.

[35] A. Kehrein and M. Kreuzer. Computing border bases. Journal of Pure and Applied Algebra, 205:279-295, 2006.

[36] A. Kehrein, M. Kreuzer, and L. Robbiano. An algebraist's view on border bases. In Solving Polynomial Equations: Foundations, Algorithms, and Applications, pages 169-202. Springer, 2005. 
[37] M. Kreuzer and H. Poulisse. Subideal border bases. preprint / arXiv:0905.1090v1, 2009.

[38] M. Kreuzer and L. Robbiano. Computational Commutative Algebra 1. Springer, 2000.

[39] M. Kreuzer and L. Robbiano. Computational Commutative Algebra 2. Springer, 2005.

[40] M. Kreuzer and L. Robbiano. Deformations of border bases. Collectanea Mathematica, 59:275-297, 2008.

[41] Martin Kreuzer. Algebraic attacks galore! Groups-Complexity-Cryptology, 1(2):231-259, 2009. doi:10.1515/GCC.2009.231.

[42] H.M. Möller. Systems of algebraic equations solved by means of endomorphisms. Lecture Notes in Computer Science, 673:43-56, 1993.

[43] B. Mourrain. A new criterion for normal form algorithms. Lecture Notes in Computer Science, 1719:430-443, 1999.

[44] Bernard Mourrain and Philippe Trebuchet. Generalized normal forms and polynomial system solving. In Proceedings of the 2005 international symposium on Symbolic and algebraic computation, pages 253-260. ACM New York, NY, USA, 2005.

[45] Bernard Mourrain and Philippe Trébuchet. Stable normal forms for polynomial system solving. Theoretical Computer Science, 409(2):229-240, 2008.

[46] S. Onn and B. Sturmfels. Cutting corners. Advances in Applied Mathematics, 23(1):29-48, 1999.

[47] J.C. Picard. Maximal closure of a graph and applications to combinatorial problems. Management Science, pages 1268-1272, 1976.

[48] S. Pokutta and A.S. Schulz. On the connection of the Sherali-Adams closure and border bases. submitted, 2009. Available from: http://www.optimization-online.org/DB_HTML/2009/08/2378.html

[49] Lorenzo Robbiano. On border basis and Gröbner basis schemes. Collectanea mathematica, 60(1):11-25, 2009.

[50] A. Schrijver. Theory of linear and integer programming. Wiley, 1986.

[51] H.D. Sherali and W.P. Adams. A hierarchy of relaxations between the continous and convex hull representations for zero-one programming problems. SIAM Journal on Discrete Mathematics, 3:411-430, 1990.

[52] L.A. Wolsey and G.L. Nemhauser. Integer and Combinatorial Optimization. John Wiley \& Sons, August 2014.

ISyE, Georgia Institute of Technology, Atlanta, GA 30332, USA.

E-mail address: gabor.brauneisye.gatech.edu

ISyE, Georgia Institute of Technology, Atlanta, GA 30332, USA.

E-mail address: sebastian.pokutta@isye.gatech.edu 